\title{
Terahertz-wave emission from Bi2212 intrinsic Josephson junctions: A review on recent progress
}

$\operatorname{AUTHOR}(\mathrm{S})$ :

Kakeya, Itsuhiro; Wang, Huabing

\section{CITATION:}

Kakeya, Itsuhiro ... [et al]. Terahertz-wave emission from Bi2212 intrinsic Josephson junctions: A review on recent progress. Superconductor Science and Technology 2016, 29(7): 073001.

\section{ISSUE DATE:}

2016-05-16

URL:

http://hdl.handle.net/2433/216928

\section{RIGHT:}

This is an author-created, un-copyedited version of an article accepted for publication in 'Superconductor Science and Technology'. The publisher is not responsible for any errors or omissions in this version of the manuscript or any version derived from it. The Version of Record is available online at http://dx.doi.org/10.1088/0953-2048/29/7/073001.; The fulltext file will be made open to the public on 16 May 2017 in accordance with publisher's 'Terms and Conditions for SelfArchiving'.; この論文は出版社版でありません。引用の際には出版社版をご確認ご利用ください。; This is not the published version. Please cite only the published version. 


\title{
Terahertz-wave emission from Bi2212 intrinsic Josephson junctions: a review on recent progress
}

\author{
Itsuhiro Kakeya \\ Department of Electronic Science and Engineering, Kyoto University, \\ Nishikyo-ku, Kyoto 615-8510, Japan \\ E-mail: kakeya@kuee.kyoto-u.ac.jp \\ Huabing Wang \\ National Institute for Materials Science, Tsukuba 3050047, Japan \\ E-mail: hbwang1000@gmail.com
}

\begin{abstract}
Emission of terahertz (THz) electromagnetic (EM) waves from a high critical temperature $\left(T_{c}\right)$ superconductor intrinsic Josephson junction (IJJ) is a new and promising candidate for practical applications of superconducting devices. From the engineering viewpoint, the IJJ $\mathrm{THz}$ source is competitive against the present semiconducting $\mathrm{THz}$ sources such as quantum cascade lasers and resonance tunnelling diode oscillators because of its broad tunable frequency range and ease of the fabrication process for the device. The emitted EM waves are considered to be coherent because the emission is yielded by synchronisation of thousand stacked IJJs consisting of the mesa device. This synchronisation is peculiar: the resonant frequency of each IJJ is distributed because the cross section of the mesa device is trapezoidal in shape. One of the key features of the synchronisation mechanism is the temperature inhomogeneity of the emitting device. In this topical review, we describe the recent progress in studies of IJJ $\mathrm{THz}$ sources with particular emphasis on the relevance of the temperature inhomogeneity to the synchronisation and the emission intensity. This review is of specific interest because the IJJ THz source shows the rich variety of functions due to self-heating which has always been a detrimental feature in the present superconducting devices. Moreover, the thermal managements used for IJJ THz sources will be common with those of other semiconducting devices such as quantum cascade lasers. In addition, this review is to invite the readers into related research through the detailed descriptions of experimental procedures.
\end{abstract}

Keywords: High- $T_{c}$ cuprate, intrinsic Josephson junction, Terahertz elecromagnetic wave, synchronization effect, self heating Submitted to: Supercond. Sci. Technol.

\section{Contents}


4 Experimental methods and emission properties $\quad 8$

4.1 Device preparation and measurement setups . . . . . . . . . . . 8

4.2 AC Josephson relation and cavity effect . . . . . . . . . . . . . 11

4.3 Emission intensity . . . . . . . . . . . . . . . . . . 15

4.4 Suitable materials . . . . . . . . . . . . . . . . . . 17

5 Thermal management of IJJ THz sources 19

5.1 Temperature inhomogeneity . . . . . . . . . . . . . . . 20

5.2 Heat manipulation . . . . . . . . . . . . . . . . . . . . 22

6 Examples of applications of terahertz EM wave sources 25

$\begin{array}{llr}7 & \text { Summary } & 26\end{array}$

\section{Introduction}

The discovery of the Josephson effect [1] in superconducting tunnel junctions illuminates the macroscopic quantum nature of superconductivity, in which the tunneling supercurrent is given by the sine of the phase difference of the macroscopic wave functions. The DC voltage across superconducting electrodes of a Josephson junction induces temporal development of the gauge-invariant phase difference between the electrodes. This was soon supported by the observation of the Fiske resonance in the presence of an external magnetic field [2]. The induced electromagnetic (EM) wave inside the tunnel barrier is established as the phase collective mode and is referred to as a Josephson plasma oscillation [3]; its frequency is given by the maximum Josephson current and the capacitance of the junction in the long-wavelength limit. The use of the Josephson plasma oscillation as an EM wave source has been a long-term quest for researchers in the field of superconductivity.

The first direct detection of EM wave radiation from single Josephson junctions was reported by Yanson et al [4] and Langenberg et al. [5] in 1965. The radiations were detected when the junction was biased on one of the Fiske steps, and the radiation powers were on the order of $1 \mathrm{pW}$ at about $10 \mathrm{GHz}$. Higher radiation powers were obtained from one- and two-dimensional arrays of synchronized Josephson junctions; for example, an array of thousands of Josephson junctions delivered a power of 160 $\mu \mathrm{W}$ at a frequency of $240 \mathrm{GHz}[6]$.

The demonstration of the intrinsic Josephson effect in $\mathrm{Bi}_{2} \mathrm{Sr}_{2} \mathrm{CaCu}_{2} \mathrm{O}_{8+\delta}(\mathrm{Bi2212})$ and other layered superconductors [7] and subsequent findings of various phenomena in these intrinsic Josephson junctions (IJJs) [8] strongly stimulated the research toward superconducting EM wave sources in the terahertz $(\mathrm{THz})$ frequency region. Since the superconducting order parameter is strongly modulated along the $c$-axis with a period of a (half) unit cell of the crystal structure of the superconductor, a single crystal can be considered as a stack of Josephson junctions consisting of superconducting electrodes and barrier layers with thicknesses of 0.3 and $1.2 \mathrm{~nm}$, respectively for Bi2212. This is in sharp contrast to a conventional Josephson junction, in which thick electrodes are connected through an oxide barrier with a thickness of less than 
$\sim 1 \mathrm{~nm}$ (several atomic layers). There are two main reasons why a stack of IJJs is suitable for use as a $\mathrm{THz}$ source. (1) A huge number of Josephson junctions are accumulated in a single crystal of Bi2212. For instance, a crystal with a thickness of $0.1 \mathrm{~mm}$ contains 70,000 junctions, which are strongly connected in series by the inductive [9] and capacitive [10, 11] couplings. The emission intensity is expected to be proportional to the square of the number of synchronous oscillators. (2) The large superconducting gap $\Delta$, which is greater than tens of meV in Bi2212 [12], protects the phase coherence from high-frequency damping. This results in the excitation of a highly coherent EM wave in the $\mathrm{THz}$ region. The theoretical upper limit of the excited EM wave is approximately $10 \mathrm{THz}$ for $\Delta \approx 50 \mathrm{meV}$. This is in sharp contrast to Josephson junctions made of $\mathrm{Nb}$ with critical temperature $T_{c}$ of $9 \mathrm{~K}$, where the superconducting gap corresponds to $0.7 \mathrm{THz}$ according to the BardeenCooper-Shrieffer theory, $\Delta=3.53 k_{B} T_{c}$.

The first milestone for the EM wave radiation from IJJs was elucidation of the Josephson plasma resonance $[13,14,15,16,17,18]$. Intensive studies of the Josephson plasma resonance revealed the damping mechanism of Josephson tunnelling [19] in cuprates and novel phase transitions in the vortex phase diagram $[20,21,22,23]$. The second milestone was the observation of various geometric effects in tiny crystals under magnetic fields parallel to the $a b$-plane. The oscillation of the $c$-axis resistivity indicates the formation of a triangular Josephson vortex (JV) lattice [24]. The Fiske steps elucidate that the DC voltage bias excites a Josephson plasma wave that propagates along a reciprocal vector of a JV lattice [25, 26]. The results suggest that in-plane magnetic fields may facilitate synchronisation among the stacked IJJs in certain cases $[27,28]$. The third and most impressive milestone is the detection of sub$\mathrm{THz}$ EM waves radiated from an IJJ mesa structure into the space outside the cryostat in the absence of an external magnetic field, which was achieved in 2007 [29] and is the main concern of this article. Subsequent research revealed a quite peculiar feature of the IJJ THz source among superconducting devices: an inhomogeneous temperature distribution. In fact, even a local temperature rise, which is always detrimental to the superconducting properties, is an essential ingredient for IJJ THz sources.

This review focuses on the relevance of the temperature distribution for high- $T_{c}$ IJJ THz sources, and is organised as follows. Section 2 describes the IJJ THz source by comparing it with other candidates for $\mathrm{THz}$ sources. Section 3 gives a brief history of Josephson plasma excitations and the emission of EM waves in IJJs. Section 4 reviews the experimental details of IJJ THz sources. Section 5 describes the state of the art technology for thermal management of IJJ THz sources. The challenges and perspectives for their practical application are discussed in Sec. 6. Finally, this article is summarised in Sec. 7 .

\section{Comparison of IJJ THz source with other $\mathrm{THz}$ sources}

In this section, the properties of an IJJ THz source are briefly reviewed to illustrate the comparison with other continuous-wave $(\mathrm{CW})$ sources such as a quantum cascade laser (QCL), and a resonance tunnel diode (RTD) oscillator. Figure 1 (a) and (b) show the operating temperature and emission power of three types of $\mathrm{THz}$ sources (IJJ THz source [30, 31, 32, 33, 34], THz-QCL [35, 36, 37, 38, 39, 40, 41], and RTD oscillator $[42,43,44,45])$ as functions of the frequency filling up the $\mathrm{THz}$ gap [46].

The most frequently investigated source is the QCL, which consists of a repeated stack of GaAs-based multiple quantum well heterostructures fabricated by molecular 


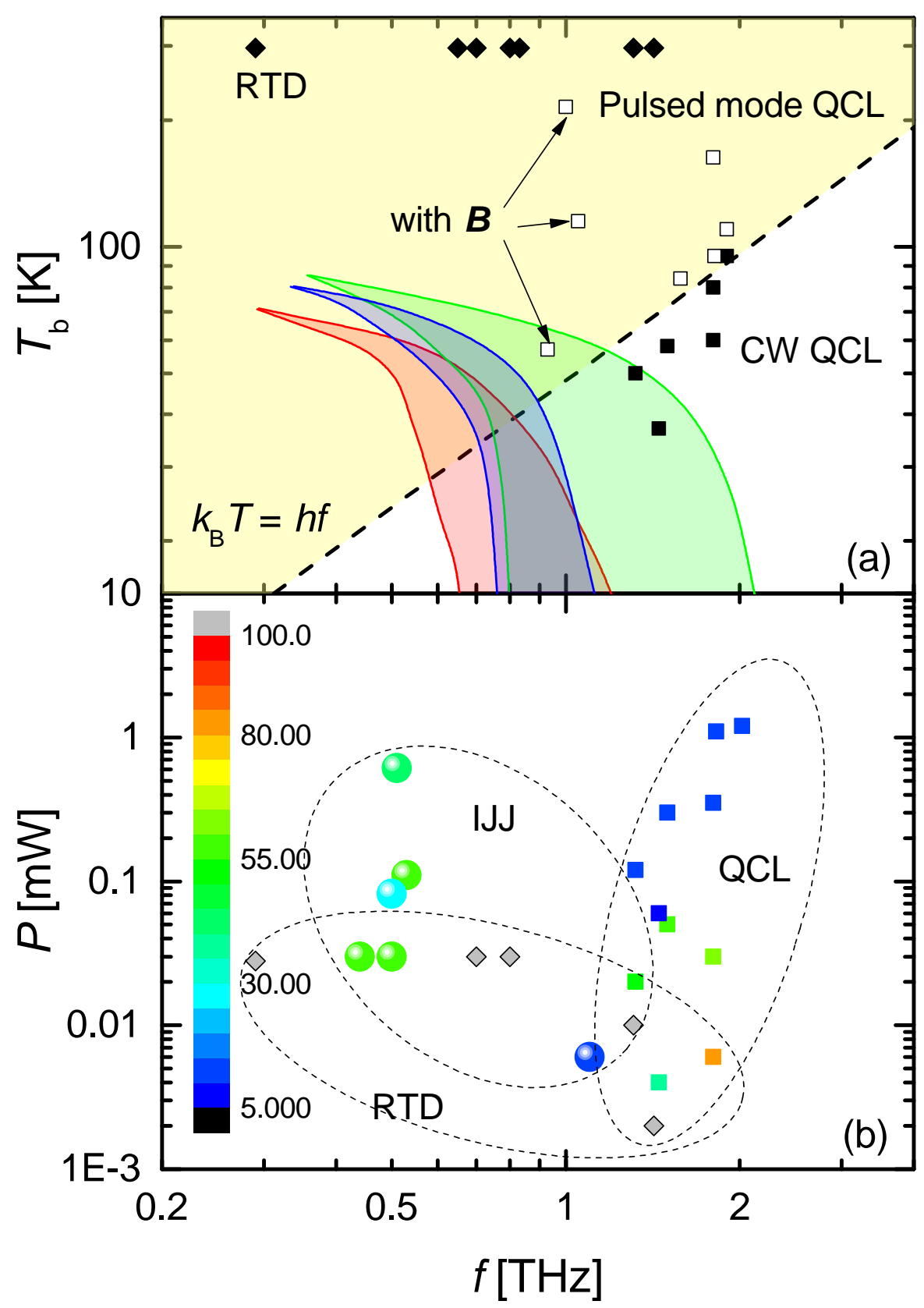

Figure 1. (a) Operation regions in temperature versus frequency of IJJ $\mathrm{THz}$ sources, in which the device structures are optimized for thermal management. A hatched area edged by solid lines represents the emission frequency region of one device. Three areas shown by red, green, and blue are obtained from the sandwich structure of a rectangular stack[33], the sandwich structure of a circular stack[34], and the gold-Bi2212-gold structure[32], respectively. Performances of CW QCLs, pulsed QCLs, and RTD oscillators are indicated by solid squares, open squares, diamonds, respectively. Data indicated by "with B" are taken from pulsed QCLs under magnetic field. (b) Maximum emission powers of IJJ THz sources (spheres), CW QCLs (squares), and RTD oscillators (diamonds). Colors of symbols represent measurement temperature for IJJ $\mathrm{THz}$ sources and CW QCLs. 
beam epitaxy [47, 48]. The emission frequency $f_{e}$ is determined by the optical transition between subbands formed in quantum well systems. Since the emission is based on population inversion between the two levels, the operating temperature is roughly limited by the thermal fluctuation; i.e., $T<h f_{e} / k_{B}$, where $h$ and $k_{B}$ are the Planck constant and Boltzmann constant, respectively $[49,50]$. In pulsed bias operation with a repetition frequency of $\sim 100 \mathrm{kHz}$ and a duty cycle of less than $5 \%$, the operating temperature significantly exceeds the upper limit up to $T>2 h f / k_{B}[36,41,50]$ as shown in Fig. 1 (a) by open squares. To extend the frequency downward to the sub- $\mathrm{THz}$ region, population inversion between two Landau levels of a two dimensional (2D) electron gas under a high magnetic field $(>5 \mathrm{~T})$ perpendicular to the layers is used [51, 38, 39]. Magnetically assisted QCLs are also operated with pulsed biases.

An RTD oscillator based on semiconductor quantum wells has been developed from operation in a lower frequency region [52]. The emission occurs in a negative differential resistance region of the device. The emission frequency is determined by the capacitance of the device and the length of the antenna and can be tuned within a few percent by varying $V$. The highest operating frequency is $1.4 \mathrm{THz}$ at a power of $1 \mu \mathrm{W}$ for devices with submicron mesas and antennas [45]. The emission power $P$ decreases dramatically with increasing frequency because of the decrease in the bias current.

In comparison with these $\mathrm{THz}$ sources, the IJJ $\mathrm{THz}$ source has following advantages:

- Since the superconducting gap protects the phase coherence from thermal damping, the emission frequency $f_{e}$ is not limited by temperature.

- The emission frequency can be easily tuned by varying the operating bath temperatures $T_{b}$ and bias voltage $V$ : the state-of-the-art maximum tuning range is $0.3-2.4 \mathrm{THz}$ [34]. This is in sharp contrast to the other two devices, the emission frequencies are determined by the device fabrication.

- Since the device function is based on the intrinsic crystal structure, highly sophisticated nanotechnologies such as molecular beam epitaxy and e-beam lithography are not required for their production. Thus, the preparation for starting research and development of IJJ $\mathrm{THz}$ sources is less demanding than those for studying other sources.

IJJ THz sources often exhibit the strongest emission around $0.5 \mathrm{THz}$ with a $T_{b}$ of 30-50 K, where QCLs do not operate in the CW mode because the population inversion collapses. The emission power is more than one-order higher than that of the RTD oscillator between 0.3 and $1 \mathrm{THz}$. The emission frequency of IJJ sources decreases with increasing $T_{b}$. Thus, the lowest emission frequency is obtained at the highest temperature, at which the active region of the device is considered to be just below the superconducting transition temperature $T_{c}$. With decreasing $T_{b}$, the emission frequency increases, and the highest $P$ is obtained at frequencies corresponding to the geometrical resonances. These phenomena are described in detail in the following sections.

\section{Electromagnetic wave excitations in IJJs}

This section briefly describes the historical background from the observation of the Josephson plasma resonance to demonstration of the $\mathrm{THz}$ emission in IJJs. 
The experimental and theoretical developments regarding EM response of IJJs are surveyed, and the distinguishing features of the discovery in 2007 are noted.

The emission of an EM wave from IJJs under a DC bias voltage is based on excitation of synchronised Josephson plasma oscillations in stacked IJJs. The Josephson plasma resonance has been investigated as a probe for Josephson and quasiparticle tunnelling and phase coherence along the $c$-axis. More detailed theoretical treatments of Josephson plasma excitation in contrast to the conventional Josephson junctions are described in Ref. [53].

The Josephson plasma resonance in layered superconductors was discovered by Tamasaku et al in $(\mathrm{La}, \mathrm{Sr})_{2} \mathrm{CuO}_{4},[13]$ and subsequently found by Tsui et al in Bi2212 [14]. Consider a spatially dependent and time-dependent gauge-invariant phase difference $\phi_{l, l+1}(x, t)$ between the $l$ and $l+1$-th $\mathrm{CuO}_{2}$ double layers of Bi2212 IJJs. Two phase collective (Josephson plasma) excitations are possible: the transverse mode uniform along the $c$-axis ( $z$-direction) and the longitudinal mode uniform over the $a b$-plane ( $x y$-direction) as shown in Figs. 2 (a) and (b), respectively. Note that the Josephson current between the $l$-th and $l+1$-th superconducting layers flows according to the DC Josephson formula as:

$$
J_{l, l+1}(x, t)=J_{c} \sin \phi_{l, l+1}(x, t),
$$

where $J_{c}$ is the maximum Josephson current density between two superconducting layers and $\phi_{l, l+1}$ is the gauge invariant phase difference between the $l$-th and $l+1$-th layers. The dispersion relation of the transverse Josephson plasma wave propagating along the $x$-axis with a wave number of $k_{x}$ is given by

$$
\omega\left(k_{x}\right)=\omega_{p} \sqrt{1+k_{x}^{2} \lambda_{c}^{2}},
$$

where $\omega_{p}=c_{0} / \sqrt{\epsilon} \lambda_{c}=\sqrt{2 e d J_{c} / \hbar \epsilon}$ and $\lambda_{c} \sim 100 \mu \mathrm{m}$ are the Josephson plasma frequency and $c$-axis penetration depth, respectively. Here, $c_{0}, d$, and $\epsilon$ denote the speed of light in vacuum, the separation between superconducting layers, and the highfrequency dielectric constant, respectively. The dispersion relation of the longitudinal Josephson plasma wave is given by

$$
\omega\left(k_{z}\right)=\omega_{p} \sqrt{1+k_{z}^{2} \mu^{2}},
$$

where $\mu \sim 1 \mathrm{~nm}$ is the charge screening length [53]. In Bi2212, $\omega_{p} / 2 \pi$ is known to be $\sim 100 \mathrm{GHz}$; thus, $\omega\left(k_{x}\right)$ can be in the THz frequency range when $k_{x}$ is on the order of $0.1 \mu \mathrm{m}^{-1}$ (a wavelength of several tens $\mu \mathrm{m}$ ) for the transverse mode.

The wavelength of an excited Josephson plasma wave inside an IJJ stack is roughly determined by its geometry, as will be discussed in Sec. 4.2. The non-linear Josephson plasma oscillation with a frequency $f$ is induced when a bias voltage $v$ is applied across an IJJ as

$$
f=2 e v / \hbar,
$$

where $e$ represent elementary charge (AC Josephson relation). Strong emission occurs when the Josephson plasma waves in hundreds of stacked IJJs are synchronous. The synchronisation mechanism of IJJs is of broad interest in physics and applied mathematics because the stacked IJJs have distributed resonance frequencies due to the trapezoidal cross sections of the stack; higher (lower) resonance frequencies are expected for upper (lower) IJJs. This system has been discussed for more than 20 years [54] as a model of various synchronization phenomena found in nature and society [55]. The detailed mechanism of excitation of synchronised transverse 

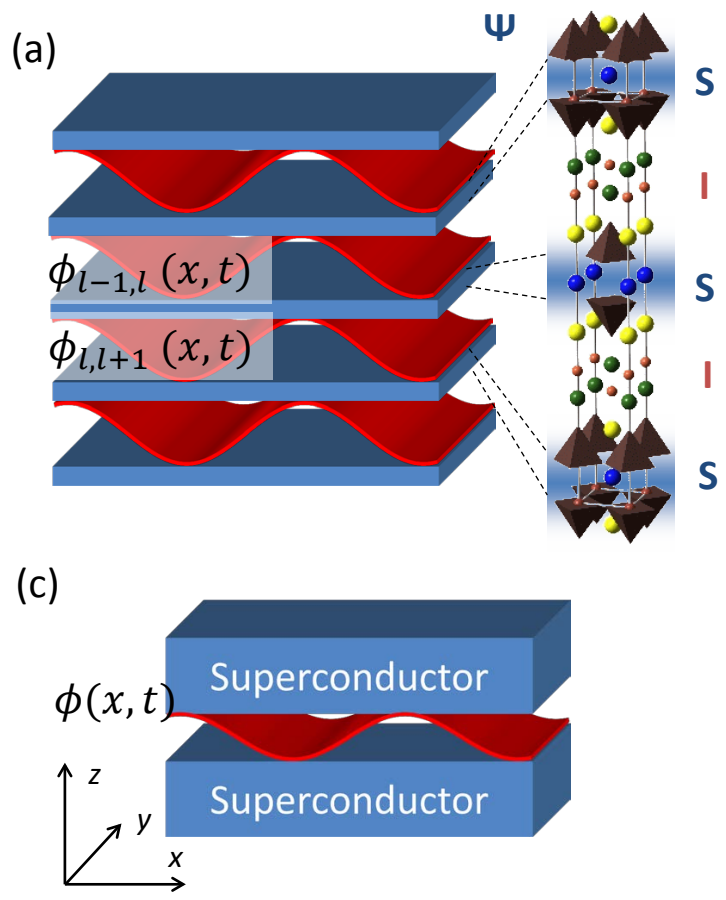

(b)
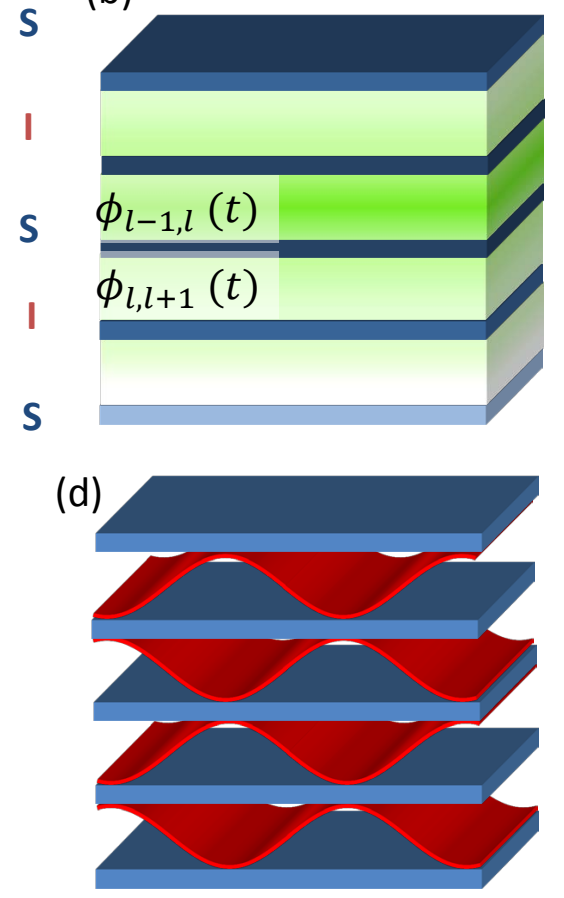

Figure 2. Schematic illustrations of (a) transverse and (b) longitudinal Josephson plasma oscillations in stacked IJJs and (d) Josephson vortex lattice induced by an external in-plane field. In (c), a single Josephson junction is depicted with the definition of directions for comparison. Wavy surfaces in (a), (c), and (d) represent gauge-invariant phase difference between adjacent superconducting layers. In (b), a blue color tone of a superconducting layer represents the charge density due to Josephon tunnelling and green gradations illustrate induced electric potential.

Josephson plasma waves is thoroughly explained in previous review articles [53, 56] and more recent papers $[57,58,59,60,61]$.

The Josephson plasma frequency $\omega_{p}$ can be measured using the Josephson plasma resonance in the long-wavelength limit by applying a uniform AC electric field along the $c$-axis over the $a b$-plane $[16,62]$. For Bi2212, the Josephson plasma resonance was experimentally investigated in the micro- and millimetre-wave regions. At $T=0$, $\omega_{p} / 2 \pi$ is in the range of $50-150 \mathrm{GHz}$ depending on the anisotropy as shown in Table 4. The Josephson plasma resonance in Bi2212 under magnetic fields parallel to the $c$-axis has been employed as a sensitive probe of the coherence between the $\mathrm{CuO}_{2}$ layers in vortex states [63]: $\omega_{p}$ shows abrupt changes accompanied by (possible) phase transitions of vortex matters [22, 23] and a higher order change in the correlations between pancake vortices defined within the $\mathrm{CuO}_{2}$ layers $[20,21,64]$ because fluctuating vortices reduce $J_{c}$ with the increase in $\phi_{l, l+1}$ according to $J_{c} \propto$ $\left\langle\cos \phi_{l, l+1}\right\rangle$, where $\langle\cdots\rangle$ represents the thermal average [15, 17, 65, 66].

EM wave absorption of the transverse Josephson plasma depending on $k_{x}$, which is the opposite process against EM wave emission, has been investigated by varying either the planar sizes of Bi2212 single crystals or the periodicity of the JV lattice 
(external field parallel to the $a b$-plane). The frequency of the transverse Josephson plasma increases with decreasing lateral sample size according to the strong dispersion relation of the transverse mode when an AC magnetic field is applied parallel to the $a b$-plane $[62,18]$. The JV lattice induced by an external magnetic field parallel to the $a b$-plane, $H_{a b}$, modulates the Josephson plasma wave along both the $c$-axis and the $a b$-plane. In the high- $H_{a b}$ region, where JVs penetrate all the block layers, the Josephson plasma frequency $\omega_{p}\left(H_{a b}\right)$ increases linearly with $H_{a b}$. This is attributed to the increase in $k_{x}$ yielded by the increase in $H_{a b}$ under the intrinsic pinning [67, 68, 69]. Theoretically, numerical simulations of EM wave radiation by flowing JVs have been developed [70,71, 72].

These experimental and theoretical evidences for coherent excitation of Josephson plasma waves inside IJJs have stimulated subsequent research for their emission. The rich variety of $I-V$ characteristics of IJJs with lateral dimensions of a few $\mu$ m under magnetic fields parallel to the $a b$-plane have been found to reveal signs of the EM wave emission [25, 26, 73, 74]. The responses of detector Josephson junctions placed in the vicinity of oscillator stacks of IJJs have shown firmer evidence for excitation of EM wave from IJJs $[75,76]$. The search for $\mathrm{THz}$ EM radiation is described in detail in Refs. [53] and [56].

The finding of Ozyuzer et al. [29] has three superiorities comparing with previous findings: (1) the large mesa with lateral dimensions of more than $50 \mu \mathrm{m}$, which is formed by easily accessible fabrication techniques without the need for sophisticated methods; (2) explicit far-field detection with an ex-situ (outside the cryostat) detector through a few windows; and (3) the absence of an external magnetic field, which yields a simple and inexpensive experimental setup.

\section{Experimental methods and emission properties}

Since the first presentation of an IJJ THz source in 2007, extensive experimental and theoretical studies have been performed. Here, we describe the experimental procedure for obtaining an IJJ THz source and the features of IJJ THz source with emphases on emission conditions, emission intensity, and material evolution. Note that most of the features of the emission frequency and spectral linewidth revealed to date are covered by previous reviews [56, 77]. More recent progress involves expanding the highest emission frequency to $2.4 \mathrm{THz}$ [34]. This topic is dealt with in the context of thermal management in Sec. 5.2.

\subsection{Device preparation and measurement setups}

Most of the IJJ $\mathrm{THz}$ devices are made of Bi2212 single crystals grown by the traveling solvent floating zone method. The mesa structures are shaped either by photolithography, metal-masked milling, or the focused ion beam (FIB) method. Among them, photolithography has been employed since this research began. The mesa and electrode patterns are designed in a photomask and are projected onto a thick $(\sim 10 \mu \mathrm{m})$ photoresist layer coated on a Bi2212 crystal glued to a sapphire or $\mathrm{MgO}$ substrate. The crystal with the shaped photoresist is milled by an Ar ion shower machine. Figure 3(a) shows a schematic image of the mesa structure on a Bi2212 single crystal obtained by this method. Hereafter, we define $x, y$, and $z$ axes as lying along the short edge in the $a b$-plane, the long edge in the $a b$-plane, and the $c$-axis stacking direction, respectively, as shown in Fig. 3. Typical dimensions are $L_{x} \times L_{y} \times L_{z}=(40-$ 
(a)

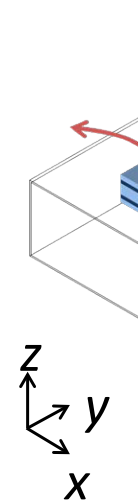

I (bias current)

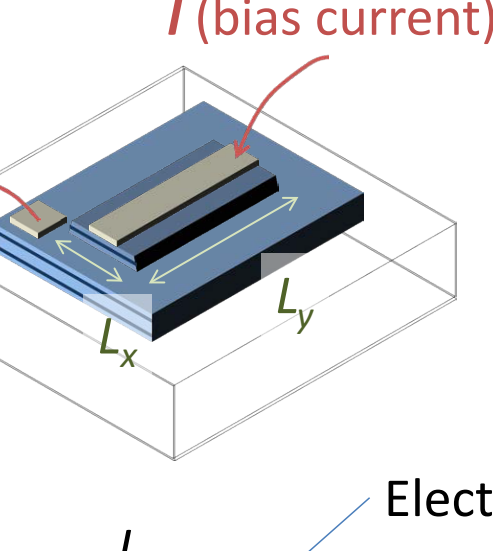

(b)
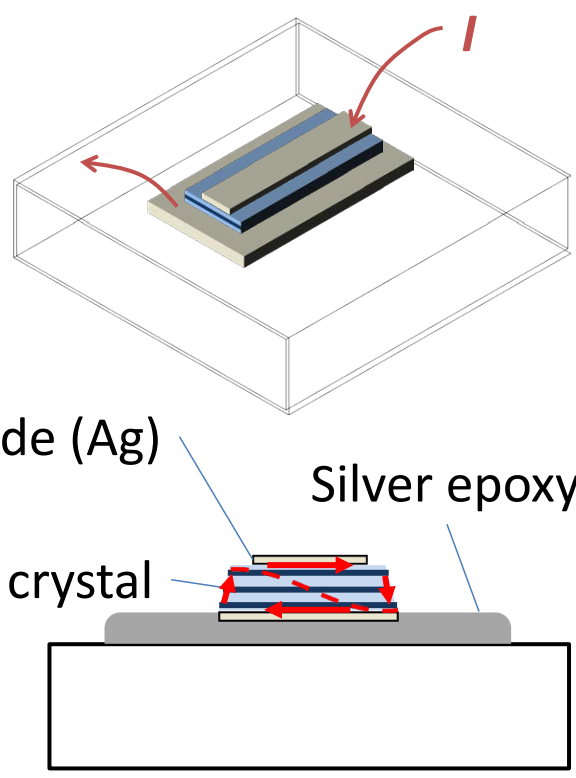

\section{Sapphire substrate}

Figure 3. (a) Mesa and (b) stand-alone devices for the THz emitter. Coordinates and dimensions are also given. In the lower panels, red broken curves represent excited Josephson plasma waves and red solid arrows represent the surface current yielding the radiation of EM waves into space.

100) $\times 300 \times 1 \mu \mathrm{m}^{3}$. Since the photoresist pattern is remarkably reduced in both thickness and area, the mesa has a trapezoidal cross section in which the length of the top surface is approximately $10 \%$ smaller than that of the bottom surface. This type of device has the disadvantages of poor heat transport and ambiguity of the number of working IJJs as discussed in Secs. 4.2 and 5. To avoid these problems, a stand-alone device was developed. In this device, the mesa is removed from the original Bi2212 base crystal and is glued to another substrate with higher thermal conductivity using a silver epoxy. For further heat elimination, sandwich structures have been reported very recently [32, 33]. Figure 4 shows the gold-Bi2212-gold (GBG) and sandwich structure (SWS), which were invented by one of the authors [32].

The metal-masked milling method employs a metal mask made by electroforming instead of using a photoresist like the photography method. This method makes it possible to have a thicker mesa because the metal mask is etched to a lesser degree by Ar ion milling than the photoresist. Because the FIB method does not require any mask, the mesa design is more flexible. In addition to the rectangular shape, circular [78], triangular [79], and pentagonal [80] shapes are formed by the FIB grooving on Bi2212 crystals. The required facilities and techniques for these and other methods are listed in Table 1. Chemical etching processes such as the hydrochloric acid modification method have the advantage of speedy fabrication of both in-line [81] and stand-alone [82] devices, although emission detection has not yet been successful. 

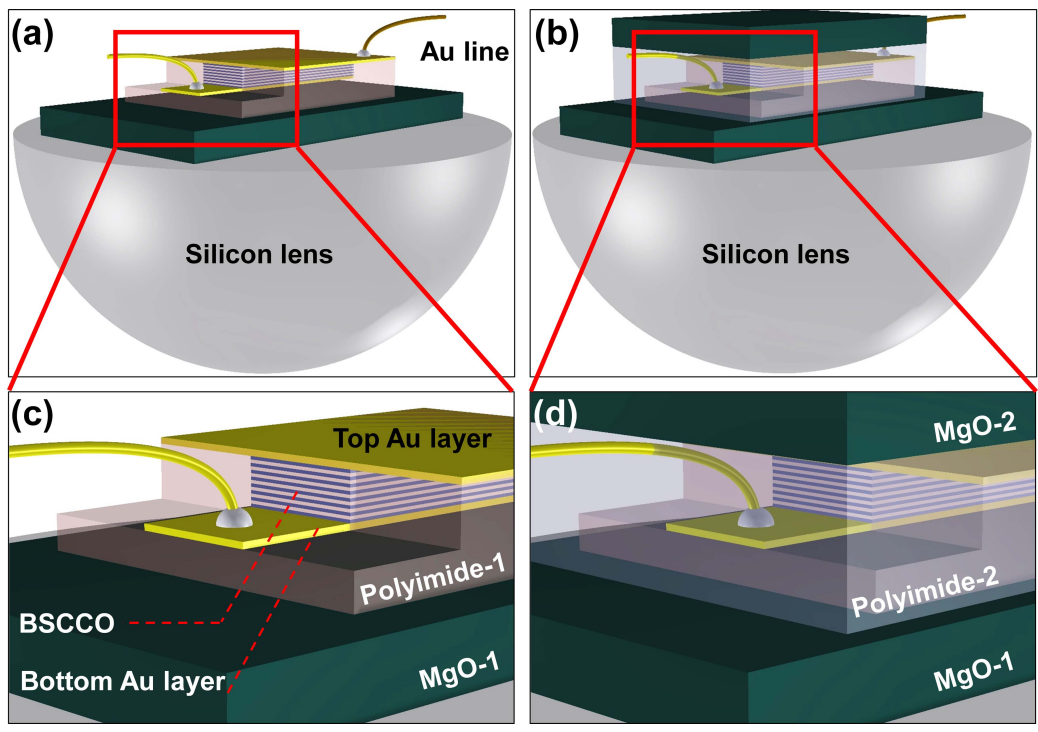

Figure 4. Schematic views of (a) GBG and (b) SWS structures mounted on the silicon lens, which were developed for improving the heat elimination. (c) and (d) show close-ups of the IJJ stacks. (after [32])

Table 1. Device preparation methods. The standard of "Ease" is ... PL, PM, $\mathrm{AL}, \mathrm{Ar}, \mathrm{EBL}$, and MM represent photolithography, photomask, alligner, Ar-ion milling machine, e-beam lithography, and metal mask, respectively.

\begin{tabular}{ccc}
\hline Method & Required techniques & Ref. \\
\hline Photolithography & PM, AL, Ar & {$[29]$} \\
PL \& Ti mask & PM, AL, Ar, EB-evapolator & {$[83]$} \\
e-Beam lithography & EBL, Ar & {$[84]$} \\
Focused ion beam & FIB & {$[78]$} \\
Metal mask & MM, Ar & {$[85]$} \\
Stand-alone & PL, FIB, MM & {$[86]$} \\
Double side & PL & {$[87]$} \\
Capped LPE & Step substrate, Furnace, PL & {$[88]$} \\
Substrate sandwich & MM, Substrate w. patterned electrodes & {$[32,33]$} \\
\hline
\end{tabular}

The most sensitive emission detection method is lock-in detection with a $\mathrm{Si}$ bolometer cooled by liquid helium. An InSb hot electron bolometer, a pyroelectric detector, and a Golay cell can also be used for higher emission power. Table 2 lists the properties of the detectors. A low-pass filter (the typical cut-off frequency was $1 \mathrm{THz}$ for most previous experiments) is inserted in front of the Winston cone to eliminate background thermal radiation. Figure 5 (a) shows a system typically used to detect EM wave emission and to determine the emission frequency. The stacked IJJs are cooled in a He-flow cryostat and are biased by either a function generator or a DC voltage (current) source. The temperature is monitored on the sample stage of the cryostat, which is regarded as a thermal bath. Since the source of the emitted EM wave is an oscillating current flowing along the short edge of the mesa, which is much shorter than the wavelength of the emitted EM wave in space, the emitted beam 
Table 2. Detectors suitable for IJJ THz sources.

\begin{tabular}{ccccc}
\hline Detector & Sensitivity & Speed & Required facilities & Ref. \\
\hline Si bolometer & Excellent & Fair & Liq. He & {$[29]$} \\
Pyroelectric sensor & Fair & Good & - & {$[89]$} \\
InSb hot-electron bolometer & Excellent & Good & Liq. He & {$[90]$} \\
Golay cell & Good & Poor & - & {$[91]$} \\
YBCO grain boundary junction & Good & Good & Inside cryo. w. emitter & {$[31]$} \\
Intrinsic Josephson junction & Good & Good & Inside cryo. w. emitter & {$[92]$} \\
\hline
\end{tabular}

diverges radially. An optical lens made of poly-crystalline Si or fused quartz placed above the mesa facilitates delivery of higher optical power to the detector.

Fourier transform infrared (FTIR) spectrometers are used to resolve the emission frequency. In addition to commercial Michelson and Martin-Puplett interferometers, as shown in Fig. 5 (b) laboratory-built interferometers are also used for spectroscopy. A simple lamellar interferometer [93] was introduced by Wang et al., as shown in Fig. 5 (c) [94]. This system does not require delicate alignment of the beam path, and most of the emitted optical power is delivered to the detector, whereas only $50 \%$ of the optical power is delivered in a Michelson-based interferometer with perfect alignment. Note that lenses are inserted, as described in the previous paragraph, to make the alignment more accurate. Turkoglu et al employed a Si single crystalline wafer as the beam splitter of a Michelson interferometer [91]. The Martin-Puplett method is suitable for attaining higher frequency resolution by extending the traveling distance of one of the reflectors. Kakeya et al. built a spectrometer with a resolution of less than $1 \mathrm{GHz}$, which is one-order higher than that of an ordinary commercial spectrometer [95].

To determine the spectral linewidth with higher frequency resolution, a heterodyne mixing method is used. Kashiwagi et al. presented a power spectrum with $\Delta f \simeq 500 \mathrm{MHz}$ for emission at the centre frequency, $f_{e}=638 \mathrm{GHz}$, at $T_{b}=10$ $\mathrm{K}$ [96]. Li et al. reported the sharpest linewidth of $23 \mathrm{MHz}$ through measurements using a superconducting integrated receiver consisting of a superconducting flux-flow oscillator and a superconductor-insulator-superconductor harmonic mixer [97].

\subsection{AC Josephson relation and cavity effect}

In the first demonstration by Ozyuzer et al., THz emission was obtained in the retrap region, where the bias voltage $V$ decreases rapidly with decreasing bias current $I$ far below the maximum Josephson current. Subsequently, Kadowaki et al. found another type of emission that is more intense and stable at a higher current bias closer to (sometimes higher than) $J_{c}$ [98]. A current-voltage $(I-V)$ characteristic and EM wave detections as functions of $I$ and $V$ of a typical device [99] are plotted in Fig. 6 (a). We refer to the latter emission as "high-bias" to distinct it from the original emission found in the "low-bias" regime. The low-bias regime corresponds to $I_{1}$ in Fig. 6 (b) and the high-bias regime corresponds to $I_{2}$ and $I_{3}$. Wang et al. also independently found $\mathrm{THz}$ emission from another IJJ device [94]. They reported for the first time that $f_{e}$ in the high-bias regime depends strongly on bath temperature $T_{b}$ and $I$. The relationship between $f_{e}$ and $V$ follows the AC Josephson relation given by Eq. (4).

The $I-V$ characteristics of the outermost (McCumber) branch of the IJJ stacks 
(a)

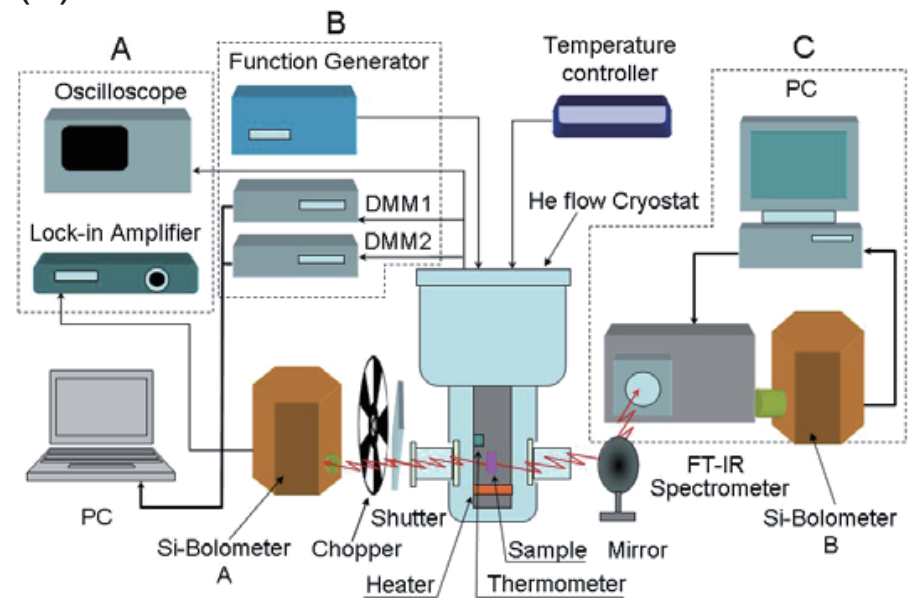

(b)

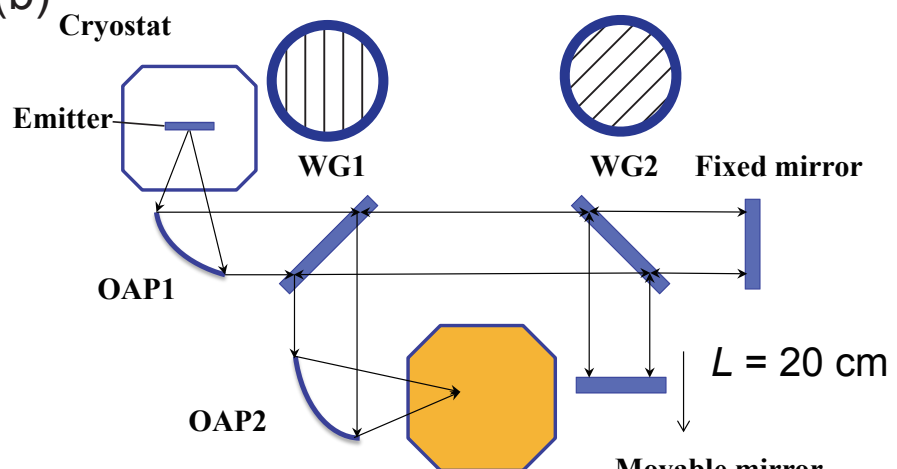

(c)

Si Bolometer

Movable mirror

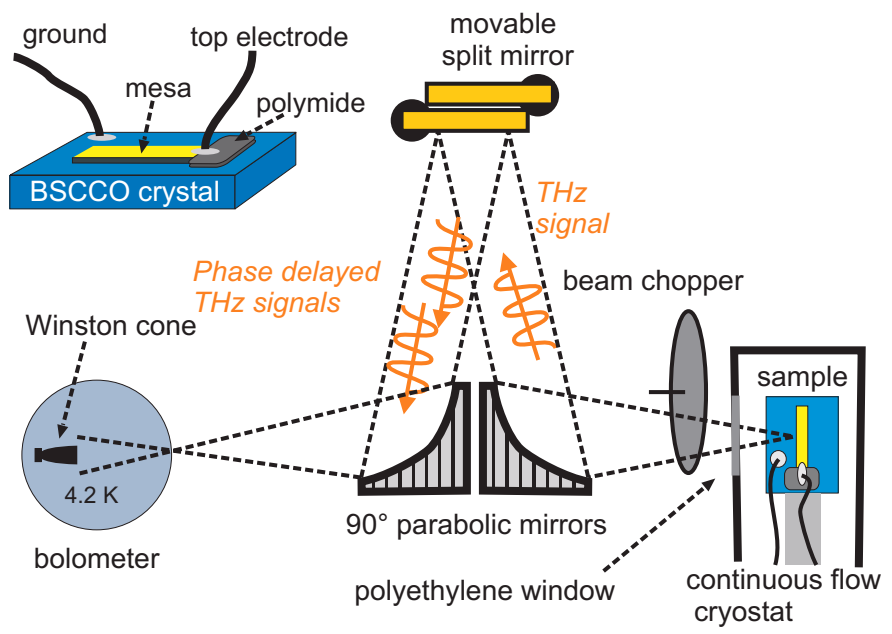

Figure 5. $\mathrm{THz}$ emission detection and spectroscopy setups. (a) An integrated system in which an emission power and an emission spectrum are simultaneously observed. (b, c) Martin-Puplet type and lamellar mirror type FTIR spectrometers. The frequency resolution can be improved by increasing the displacement between two mirrors. (after [98], [95], and [94], respectively) 
found in the downward current sweep are remarkably nonlinear as illustrated in Fig. 6 (b) because of the following reasons. At bias currents sufficiently higher than the critical current (above $I_{3}$ ), the device temperature may be more than 10 Kelvins higher than $T_{b}$ because of self-heating of the device and poor thermal conductivity of Bi2212. Since the $c$-axis resistivity of Bi2212 IJJs in the normal state is expected to maintain the rapid increase with decreasing temperature even below $T_{c}$, the decrease in the device temperature due to the decrease in $I$ causes an increase in $P$ below $I_{2}$. A further decrease in $I$ restores the zero-resistance of IJJs. Note that the $c$-axis bias current accumulates at which the local temperature is higher (local resistance is lower) and this accumulation makes the non-linearity of the $I-V$ characteristics more significant. The local temperature rise above $T_{c}$ is referred as a "hotspot" and is discussed in Sec. 5.1. The resultant S-shaped $I-V$ curve as shown in Fig. 6 (b), provides up to three bias currents $I_{1}, I_{2}$, and $I_{3}$ with the same $V$, which induces an EM wave with $f=2 e V_{b} / N h$ according to the AC Josephson relation (Eq. (4)), where $N$ is the number of IJJs in voltage state. The emission occurs when $f_{e}$ matches to a cavity resonance condition as described in the following paragraphs.

The emission phenomena in the low-bias regime $\left(I_{1}\right)$ are less controversial than those in the high-bias regime $\left(I_{2}\right.$ and $\left.I_{3}\right)$. The emission frequency is primarily explained by a matching effect between the AC Josephson effect and the cavity resonance of the $\operatorname{TM}(1,0)$ mode in the mesa structure [29]. The emission is less stable because some of the stacked IJJs return to zero-resistance (retrapping) in this current region, resulting in a sudden voltage drop with decreasing current. On the other hand, the high-bias regime exhibits a rich variety of phenomena such as broad-band frequency tuning, significant local temperature increases, and surprisingly narrow spectra $[98,100,94,97]$. The directionality and duration of continuous emission were initially tested for high-bias emission, providing relevant intuition for excitation inside the mesa [89]. A few reports mention emission at the highest bias currents corresponding to $I_{3}[101,102]$. The emission frequency in this regime is significantly lower than that predicted by the AC Josephson relation [102]. This is explained by a significant temperature gradient along the stack (c-axis): uniformly hotter layers near the surface determine the emission frequency, whereas colder layers near the bottom contribute to the excess voltage.

The cavity model gives the frequency of the excited transverse Josephson plasma wave inside a rectangular IJJ stack shown in Fig. 3 (a) as follows:

$$
f_{m, p}^{c}=\frac{c_{0}}{2 n} \sqrt{\left(\frac{m}{L_{x}}\right)^{2}+\left(\frac{p}{L_{y}}\right)^{2}},
$$

where $n$ is the refractive index of the mesa, and $m$ and $p$ represent the number of nodes of the standing wave along the $x$ and $y$ directions, respectively. In a mesa with a large aspect ratio $\left(L_{x} \ll L_{y}\right)$, since the emission frequency is proportional to $1 / L_{x}$, primarily the $\operatorname{TM}(1,0)$ mode is excited. The details are described in Sec. 5.1. In a mesa with an aspect ratio close to unity, we often observe emissions attributed to various cavity modes $\operatorname{TM}(m, p)$. The agreement with experimentally observed emission frequencies is summarised in Fig. 7. However, it is still difficult to unambiguously determine the cavity mode because the emission intensity is rather continuous with respect to the frequency owing to the degeneracy in frequencies of different modes [86]. Even mesas with circular [78], triangular, and pentagonal [79] shapes emit not only with certain cavity modes but also with undefined modes. Quite recently, excellent 
(a)

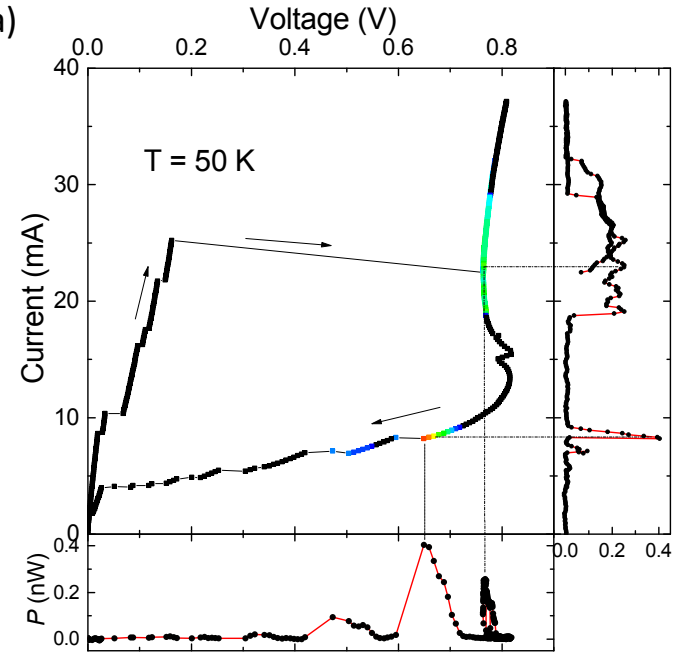

(b)

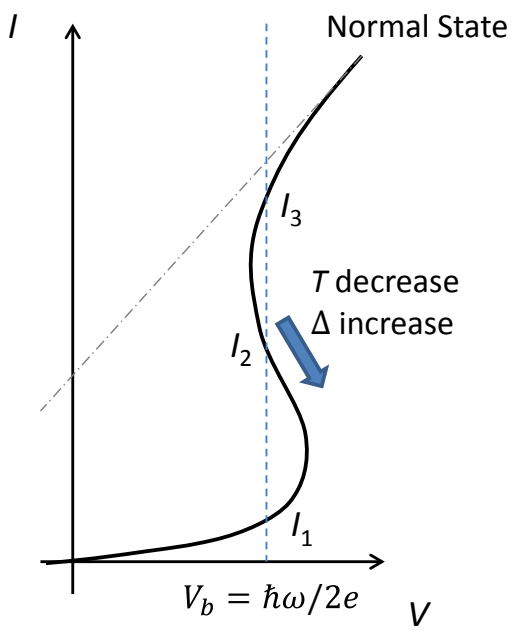

Figure 6. (a) $I-V$ characteristics (main) and bolometer response (bottom and right) for an IJJ $\mathrm{THz}$ sources at $T_{b}=50 \mathrm{~K}$. Arrows indicate scanning directions. With increasing $I, V$ jumps from 0.17 to $0.77 \mathrm{~V}$ at the critical current approximately $25 \mathrm{~mA}$. Then the device starts to emit EM wave. With decreasing $I$ from its maximum, $V$ traces a different path below the critical current. EM wave emissions are observed at high-bias region $(18<I<30 \mathrm{~mA})$ and low-bias region $(6<I<10 \mathrm{~mA})$. Discontinuities of the $I-V$ curve below $10 \mathrm{~mA}$ starting at the low-bias region are due to the retrapping (see text). (b) Schematic illustration of the S-shape behaviour in an $I-V$ curve of the outermost branch. With decreasing bias current, the device temperature decreases, and then the superconducting gap $\Delta$ increases. In the higher current limit (normal state), the $I-V$ curve should asymptote to the Ohmic line indicated by a broken line.

correspondence between emission peaks and cavity modes was reported throughout numerical calculations [103].

For tuning emission frequency $f_{e}$ of one device in-situ, either $n, L_{x}$, or $L_{y}$ has to be modified effectively by tuning bias conditions and $T_{b}$. The wide-range tuning of $f_{e}$ of the IJJ THz source arises effective variations in $n, L_{x}$, and $L_{y}$ due to change of device temperature. The effective $n$ depends on device temperature through the temperature dependence of the London penetration depth $\lambda_{L}$ in the presence of the longitudinal component of an excited Josephson plasma oscillation (finite $k_{z}$ ) [104]. With increasing device temperature, $f_{e}$ decreases through the decrease in propagating speed of the Josephson plasma oscillation due to the increase in $\lambda_{L}$. The increase of $I$ raises device temperature, also resulting in the decrease of $f_{e}$ [105]. The effective changes of $L_{x}$ and $L_{y}$ are discussed in Sec. 5.1.

The emission power $P$ was found to be much more intense in the $z$-direction than in the $x$-direction [98], although the first observation was monitored only in the $x y$-direction [29]. This result suggests that the alternating current induced by the transverse Josephson plasma oscillation appears not only on the $y z$-surface but also on the $x y$-surface of the mesa. The alternating current is inductively coupled with the space, resulting in a propagating EM wave polarised along the $x$ axis. For the $\operatorname{TM}(1,0)$ mode, the emission intensity is greatest in the direction tilted by $20-30^{\circ}$ from the $z$-axis toward the $x y$-plane, according to directionality measurements in the 


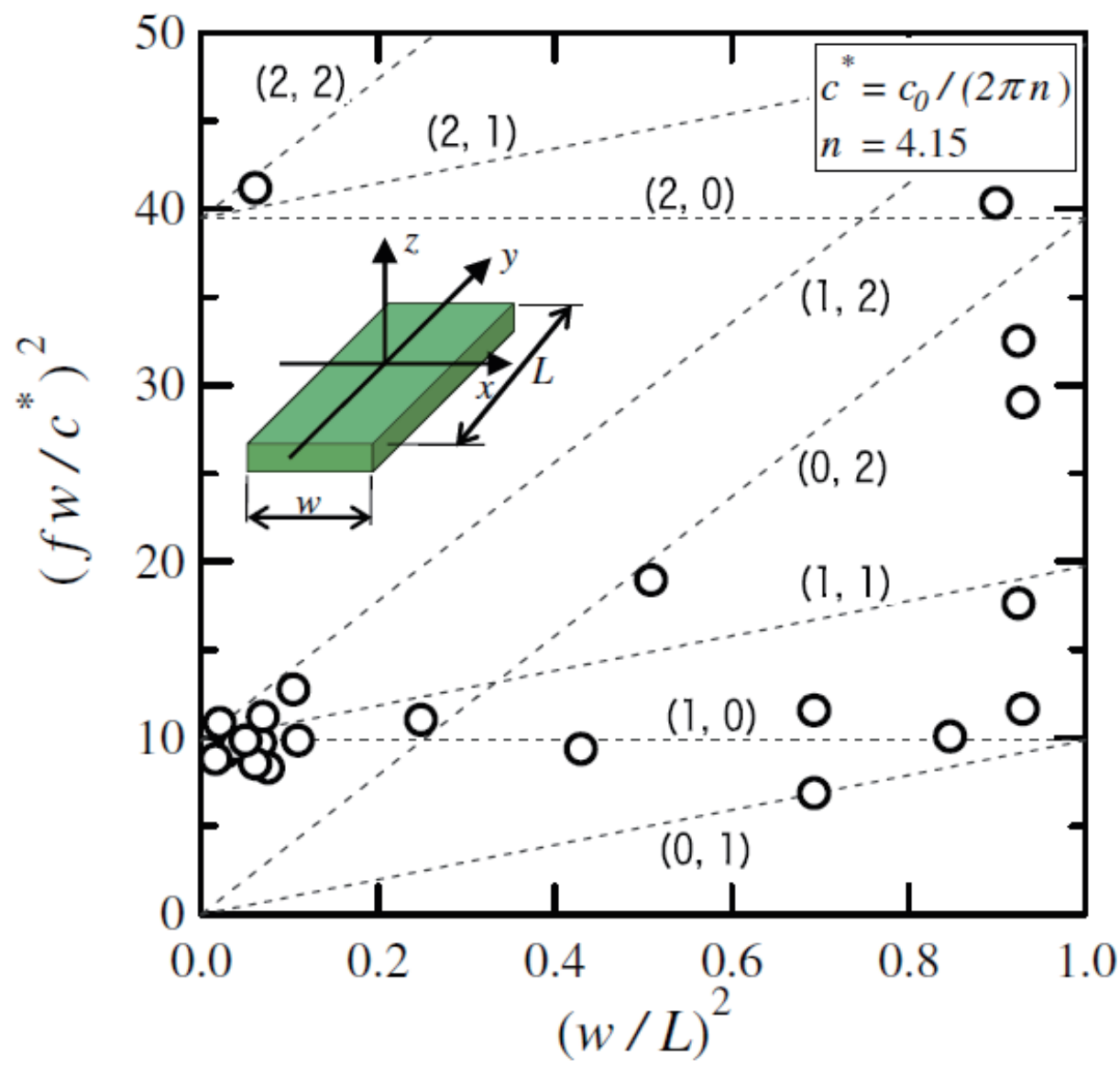

Figure 7. Plot of the typical emission frequencies vs several cavity resonance modes. $c_{0}$ is the speed of light in vacuum, and $n$ is the refractive index of the material. $w$ and $L$ correspond to $L_{x}$ and $L_{y}$ in the present review, respectively. (after [96])

$x z$-plane. [89] This is attributed to interference between a wave coming directly from the mesa and a wave reflected by the superconducting substrate. For an asymmetric device in which a mesa is placed at an apparently off-centred position on the substrate, the directionality becomes asymmetric [106].

\subsection{Emission intensity}

The emission intensity is one of the most important parameters describing the performance of an EM wave source. To date, a maximum total emission power of $P_{\text {tot }}=610 \mu \mathrm{W}$ has been achieved by synchronising three IJJ stacks at $T_{b}=40 \mathrm{~K}$ [106]. Note that direct comparison of the emission (detection) power among groups and comparison with other $\mathrm{THz}$ sources are difficult because the total emission power $P_{\text {tot }}$ is estimated assuming losses in the optical components, and the detected power $P$ sometimes represents the beam power focused by a lens. Table 3 summarises the 
Table 3. Summary for emission powers. BM, PM, and GBJ represent bolometer, powermeter, and grain-boundary junction, respectively.

\begin{tabular}{cccccc}
\hline Device type & reported power & Detector & Estimation & Note & Ref. \\
\hline Single mesa & $30 \mu \mathrm{W}$ & Si-BM, InSb-BM, PM & directionality in two planes & Integ. pow. & {$[107]$} \\
Sync. 3 mesas & $610 \mu \mathrm{W}$ & Si-BM & calib. by a black-body source & Integ. pow. & {$[106]$} \\
Single mesa & $110 \mu \mathrm{W}$ & & & & {$[106]$} \\
Double-sided & $82 \mu \mathrm{W}$ & Si-BM, YBCO GBJ & & Direct detection & {$[32]$} \\
\hline
\end{tabular}

maximum emission powers reported by various groups [31, 106, 107].

The first reliable estimation of $P_{t o t}$ considering the directionality was $5 \mu \mathrm{W}$ [89], and a higher $P_{t o t}$ of $30 \mu \mathrm{W}$ was subsequently reported by Yamaki et al. [108] More exact estimations were recently presented by Sekimoto et al. [107]. They reported a $P_{t o t}=30 \mu \mathrm{W}$ after careful calibration of their detection setup and emission directionality as shown in Fig. 8. An InSb hot electron bolometer calibrated by a blackbody radiation source was used for detection, in addition to a Si bolometer and a commercial power meter. The results obtained from the three different detectors show good consistency.

To increase the emission power, synchronisation of multiple mesas has been attempted since the early research. The pioneering result was obtained by Orita et al. [85] Two mesas with slightly different emission frequencies (0.68 and $0.70 \mathrm{THz})$ were electrically connected in series and driven simultaneously. As a result, the two mesas emitted EM waves at another frequency $(0.65 \mathrm{THz})$, and the maximum $P$ significantly exceeded the sum of the individual emissions. Benseman et al. were able to detect the maximum power emitted from an IJJ device, which is $P_{t o t}=610 \mu \mathrm{W}$ at $510 \mathrm{GHz}$ by synchronising three mesas on a Bi2212 single crystal biased in parallel as shown in Fig. 9 [106]. They carefully calibrated their detection system with a standard black-body radiator and managed the huge amount of dissipated from a crystal soldered onto a metallic copper substrate. The emitted EM waves were monochromatic up to the resolution limits of their spectrometers; thus, the EM waves excited in the individual mesas are synchronised.

The stand-alone devices illustrated in Fig. 3(b) were originally invented to increase the emission power. These attempts were based on a theoretical suggestion that the image super-current flowing in the superconducting substrate underneath the mesa diminishes the emission power [109]. Although the early preparation procedures for the stand-alone devices were technically complicated [96, 110, 105], Kashiwagi et al., developed an easier procedure to obtain systematic results [111]. The emission power from stand-alone devices tends to be higher than that from conventional mesa devices [110]. However, the direct contact of the mesa with the substrate facilitates thermal dissipation into the substrate rather than removal of the image current. An et al.[31] combined a stand-alone $\mathrm{Bi}_{2} \mathrm{Sr}_{2} \mathrm{CaCu}_{2} \mathrm{O}_{8}$ IJJ stack, emitting $\mathrm{THz}$ radiation, with a $\mathrm{YBa}_{2} \mathrm{Cu}_{3} \mathrm{O}_{7}$ grain boundary Josephson junction acting as a detector. With the emitter radiating at $0.5 \mathrm{THz}$, they observed up to seven Shapiro steps in the $I-V$ characteristics of the detector. The AC current induced in this detector junction was $I_{a c}=0.93 \mathrm{~mA}$, which indicates that the power picked up by the antenna is $P=I_{a c}^{2} Z / 2 \approx 82 \mu \mathrm{W}$, where the impedance of the antenna $Z \approx 189 \Omega$. 


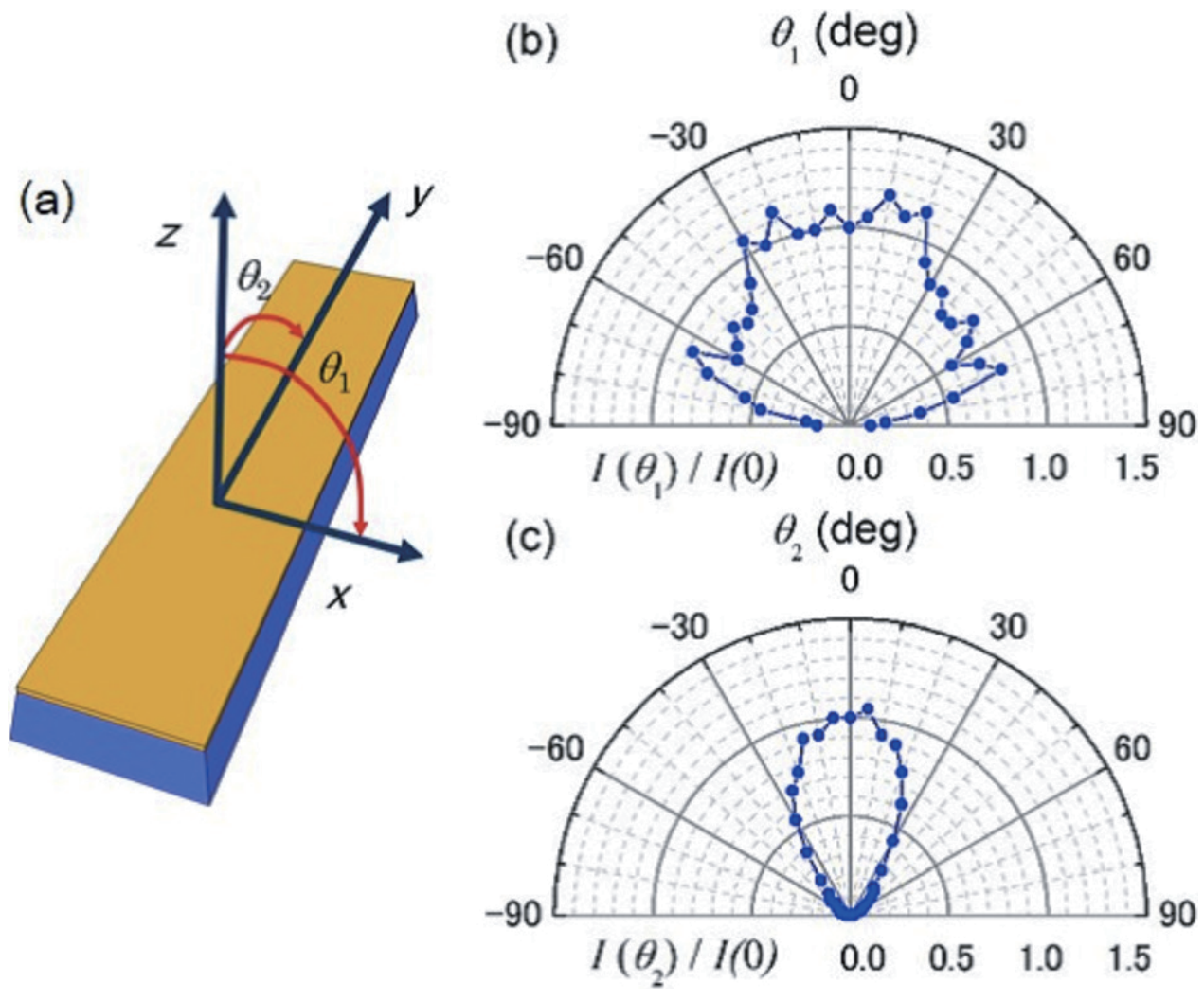

Figure 8. (a) The definitions of $\theta_{1}$ and $\theta_{2}$. (b) Polar plots of the radiation intensity $I\left(\theta_{1}\right)$ normalized at $I(0)$ measured in the $x z$-plane at $55 \mathrm{~K}$. (c) Polar plots of $I\left(\theta_{2}\right)$ normalized at $I(0)$ measured in the $y z$-plane at $55 \mathrm{~K}$. (after [107])

\subsection{Suitable materials}

It is well-known that the $T_{c}$ value of high- $T_{c}$ cuprates depends strongly on the carrier doping $p$, where $T_{c}$ exhibits a dome-shape as a function of $p$ [112]. The carrier doping also changes the IJJ properties; with decreasing $p, J_{c}$ decreases exponentially, the quasiparticle tunnelling resistivity increases, and phase diffusion is less pronounced. Because the THz emission from IJJs was first demonstrated in strongly under-doped Bi2212 with $T_{c} \simeq 70 \mathrm{~K}$, an under-doped crystal is believed to be more suitable for $\mathrm{THz}$ emission. Certainly, a higher $V$ due to the higher quasiparticle resistivity gives rise to a higher $f_{e}$ satisfying the Josephson relation [Eq. (4)]. Moreover, the lower retrapping current when the IJJ returns to the superconducting state, which occurs because there is less phase diffusion (damping), expands the voltage region in which the emission condition is satisfied, especially for low-bias emission. Nevertheless, it has been found that under-doping is not essential for emission. Intensive emission is reported in crystals with higher $J_{c}$ values and $T_{c} \simeq 90 \mathrm{~K}$. Figure 10 plots $f_{e}$ versus the critical current density $J_{c}$, where the symbol size and colours represent $L_{x}$ and the measured $T_{b}$, respectively. $f_{e}$ is obtained from the spectrum with the maximum peak height among spectra taken under various bias voltages and temperatures for a 

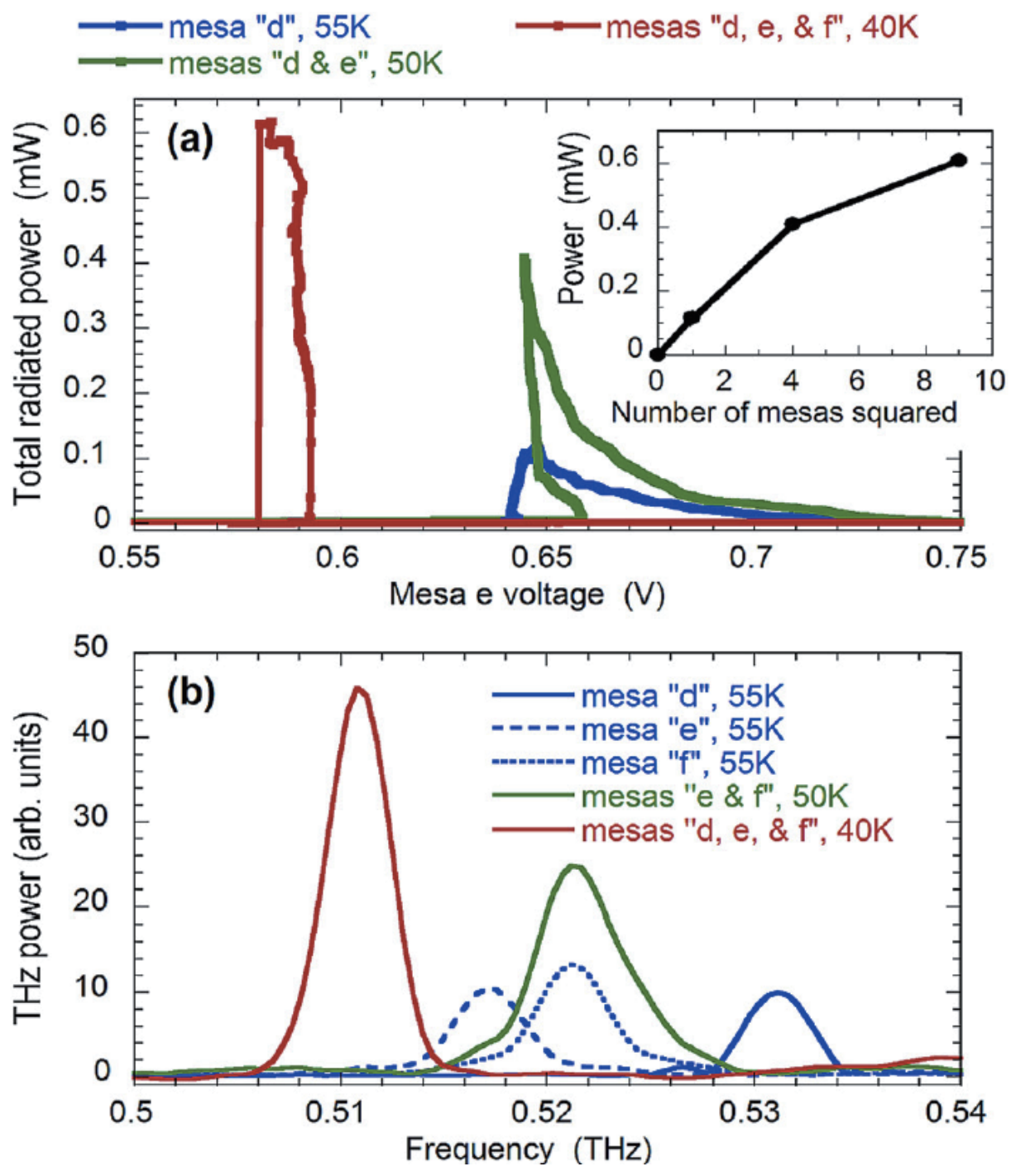

Figure 9. (a) Bias dependence of the $\mathrm{THz}$ emission power $P_{t o t}$ of an array of mesas for various activated mesas and the maximum power as a function of the number of activated mesas and (b) emission spectrum of the array for various activated mesa. (after [106]) 


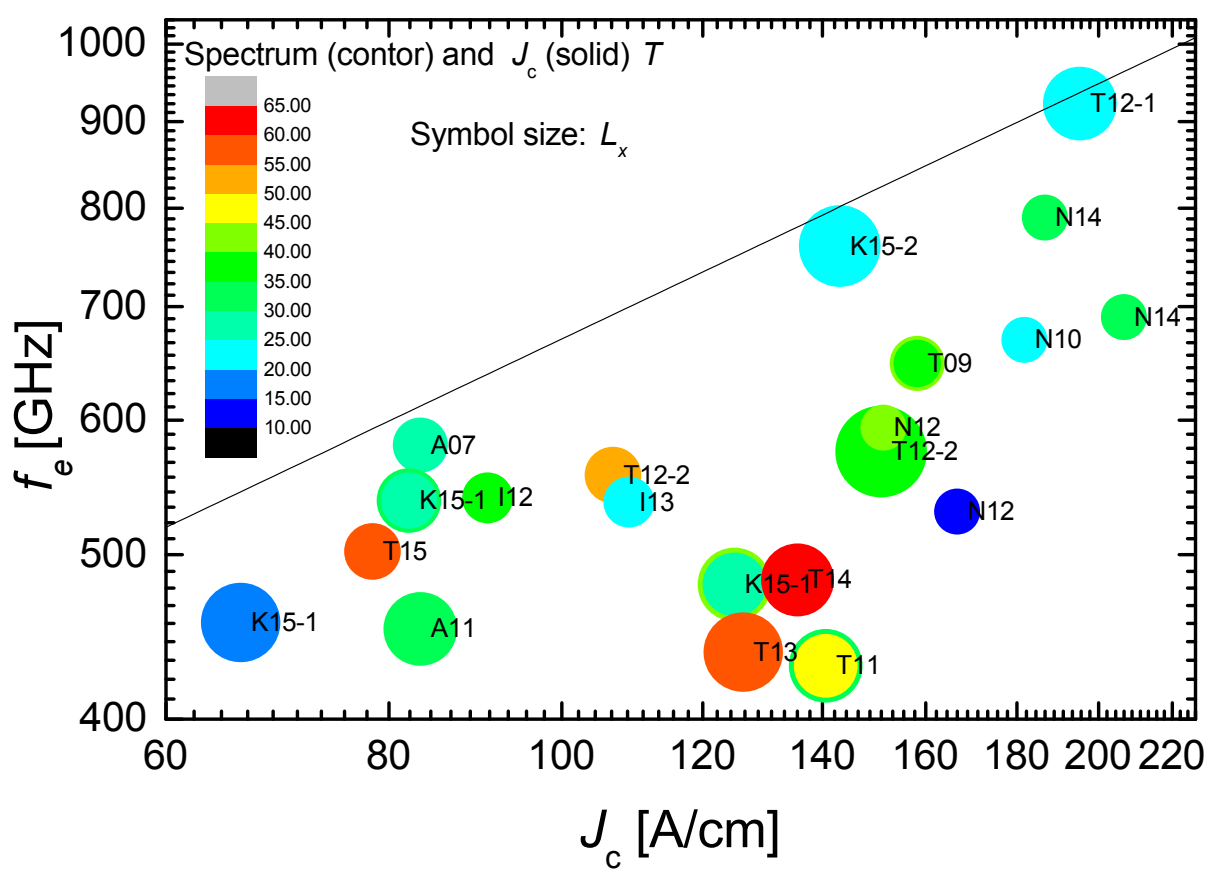

Figure 10. Emission frequencies as a function of $J_{c}$. Symbol sizes and colours represent $L_{x}$ and measured temperatures. A solid line represents $f_{e} \propto \sqrt{J_{c}}$. Data correspondences are A07 [29], A11 [104], I12 [91], I13 [113], K15-1 [105], K152 [114], N10 [94], N12 [87], N14 [32], T09 [89], T12-1 [96], T12-2 [86], T14 [110], T15-1 [33], and T15-2 [34].

specific device. The upper limit of the optimised frequency appears to depend on $J_{c}$ rather than $L_{x}$ and roughly follows $\sqrt{J_{c}}$, which is the same evolution as the Josephson plasma frequency $\omega_{p}$.

Very recently, emission from Bi2212 substituted materials has been reported. Tsujimoto et al found emission from $\mathrm{Pb}-\mathrm{Bi} 2212$ stacks with a $J_{c}$ value three times larger than that of usual pristine Bi2212 mesas [114]. To suppress the temperature rise, a stand-alone device was used. The emission frequency was found to be almost twice that expected in a pristine Bi2212 device with the same dimensions. The effective refractive index is presumably smaller in $\mathrm{Pb}-\mathrm{Bi} 2212$. More recently, emission was also found in La-Bi2212 which is expected to have less damped IJJs. Material evolution has great potential for the functionalisation of IJJ THz sources because the junction parameters can be tuned over a wide range. Related parameters of IJJ materials are listed in Table 4

\section{Thermal management of IJJ THz sources}

In most experiments to date, the emission frequencies were well below the frequency corresponding to the superconducting gap energy of Bi2212. The main obstacle to emission at higher frequencies is self-heating, which limits the maximum voltage $V_{\max }$ across the stack. Self-heating is more severe when there are more junctions 
Table 4. Reported dielectric constants $\epsilon$ and Josephson plasma frequencies $\omega_{p} / 2 \pi$ of IJJ materials. " $\rightarrow 0$ " represents extrapolation to $0 \mathrm{~K}$.

\begin{tabular}{cccccc}
\hline Material & $T_{c}[\mathrm{~K}]$ & $\epsilon$ & $\omega_{p} / 2 \pi[\mathrm{GHz}]$ & $T[\mathrm{~K}]$ & Ref. \\
\hline $\mathrm{Bi} 2212$ & 70.2 & & 56.8 & $\rightarrow 0$ & $T$-scan at various $\omega[69]$ \\
$\mathrm{Bi2} 212$ & 68.0 & & 68 & $\rightarrow 0$ & $\omega$-scan [19] \\
$\mathrm{Bi2212}$ & 82.5 & & 124 & $\rightarrow 0$ & $\omega$-scan [19] \\
$\mathrm{Bi2212}$ & 90 & 12 & & 6 & FTIR [115] \\
$\mathrm{Bi} 2212$ & 85 & & 150 & 5 & FTIR [116] \\
$\mathrm{Bi} 2212$ & 71 & & 330 & 5 & FTIR [116] \\
$\mathrm{Bi}_{1.6} \mathrm{~Pb}_{0.6} \mathrm{Sr}_{1.8} \mathrm{CaCu}_{2} \mathrm{O}_{8+\delta}$ & 65 & 11.5 & 1150 & 8 & FTIR [117] \\
$\mathrm{La-Bi2212}(p=0.168)$ & 85 & 6 & 186 & 20 & $19 \mathrm{GHz}, 30 \mathrm{kOe}[118]$ \\
$\mathrm{Bi} 2201$ & 16.5 & & 67 & $\rightarrow 0$ & $\omega$-scan [19] \\
$\mathrm{Nd}_{1.85} \mathrm{Ce}_{0.15} \mathrm{CuO}_{4}$ & 18.0 & 14 & 174 & 11 & T-scan at fixed $\omega[119]$ \\
$\mathrm{Tl}_{2} \mathrm{Ba}_{2} \mathrm{Ca}_{2} \mathrm{Cu}_{2} \mathrm{O}_{8}$ & 103.4 & 9.1 & 780 & 10 & THz-TDS [120] \\
\hline
\end{tabular}

in the stack and in stacks with larger lateral sizes. As a result, we typically observe severe bending of the $I-V$ curves at high bias currents or voltages. The strong temperature inhomogeneity has been studied experimentally and theoretically $[100,102,121,122,123,124,125]$

\subsection{Temperature inhomogeneity}

The first representation of temperature inhomogeneity was reported using the LTSLM [100]. The principle of the LTSLM is described in the supplementary material of Ref. [100]. In short, the beam of a diode laser with a spot size of $1-2 \mu \mathrm{m}$ is deflected by a scanning unit and focused onto the sample surface. Local heating by $2-3 \mathrm{~K}$ in an area of a few $\mu \mathrm{m}^{2}$ and about $0.5 \mu \mathrm{m}$ in depth causes a change in the voltage across the mesa, $\Delta V$, which is detected using lock-in techniques, and serves as the contrast of the LTSLM image. Standing waves can also be imaged by using a local change in the quality factor induced by the laser beam. This results in a stronger $\Delta V$ signal at antinodes and a weaker signal at nodes.

Recently, a mini-LTSLM was developed on the centimetre scale [126]. A schematic of the mini-LTSLM is shown in Fig. 11. A sample is mounted on a hemispheric sapphire lens. A laser with wavelength $\lambda=1310 \mathrm{~nm}$ and a spot diameter of about $1 \mu \mathrm{m}$ is focused onto the surface of the stack. The laser can scan across the surface of the stack with submicron precision. Thanks to the small size of the setup which includes a Si bolometer, it can also measure the emission power of the sample from the other side of the hemispheric lens while the laser is kept on at the same time [126]. The power detection and frequency measurement are similar to the previous work [127]. By modulating the laser power using a $10 \mathrm{kHz}$ square signal, we detect the global differential voltage response $\Delta V$ of the stack with a lock-in technique while the sample is biased at a constant current. In this case, the setup serves as an LTSLM [100, 94, 127, 128]. Recently, Zhou et al. used a relatively strong beam power to operate the LTSLM in a manipulation mode at the same time.

Joule heating in large Bi2212 mesas was numerically analysed by considering the typical terminal conductivity and the temperature dependence of all the materials associated with heat dissipation and removal. Such mesas are used in experiments on THz-range radiation. The analysis shows that the temperature increases with 


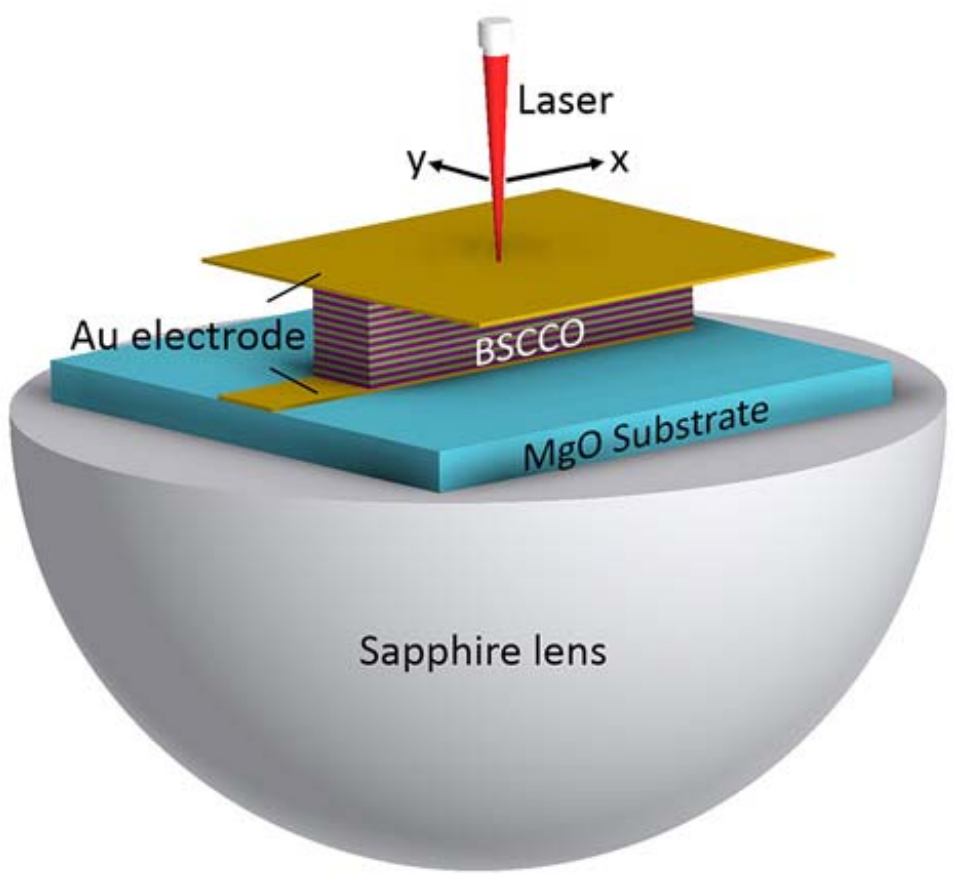

Figure 11. Sketch of mini-LTSLM system for the GBG structure sample on a hemispheric sapphire lens. A focused laser can scan across the sample surface while emission is detected through the lens by a Si bolometer (not shown in the sketch). This system allows us to measure simultaneously both changes in voltage of the device and emission power due to the focused laser irradiation. (after [126])

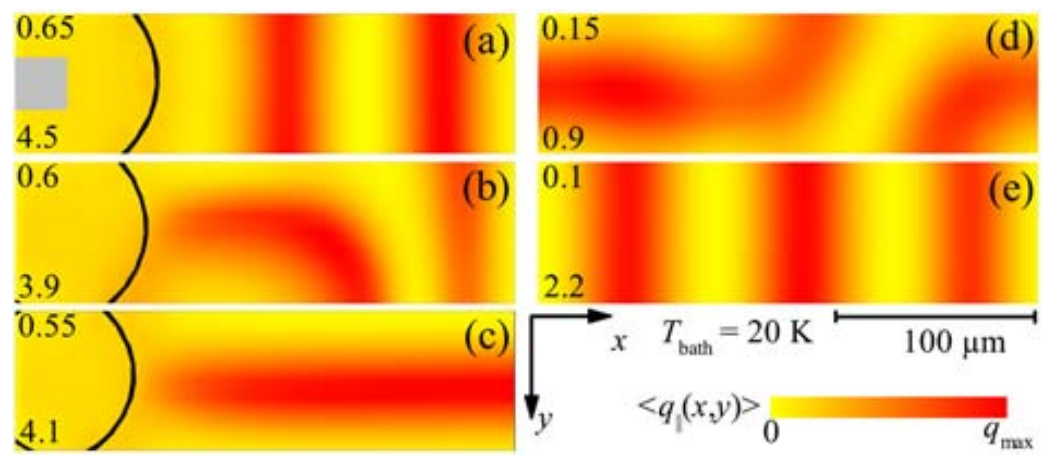

Figure 12. Power density dissipated by in-plane currents for five values of normalized bias current at upper left and maximum dissipation current at bottom left. The grey square in (a) indicates position of the bond wire. Black curves are drawn where the local temperature corresponds to $T_{c}$ and the regions closer to the injection point are at temperature higher than $T_{c}$. (after [125]) 
increasing $I$ and is distributed unevenly along the mesas. The temperature of the mesa's central part can even exceed $T_{c}$ at sufficiently high bias, forming a hotspot. The overall $I-V$ characteristics are also calculated self-consistently and show a negative differential conductance for a wide range of currents [121]. Gross et al. reported similar results on hotspot formation in stacks of IJJs in Bi2212 [128]. The formation of hotspots in this system is shown to arise mainly from the strongly negative temperature coefficient of the $c$-axis resistivity of Bi2212 at low temperatures.

Quite recently, Rudau et al. used 2D coupled sine-Gordon equations combined with three-dimensional heat diffusion equations to numerically investigate the thermal and EM properties of a 700-IJJ stack [125]. Typical numerical simulation results are shown in Fig. 12. A strong field -induced enhancement of $P$ by a factor of almost 3 was found experimentally, for an applied field of around $5.9 \mathrm{mT}$. Although the numerical simulations are in good agreement with the experimental observations, direct measurement of the temperature is required to confirm these experimental and theoretical results.

Two fluorescence methods have been employed to monitor local variation in the mesa temperature: coating the device with a polymer film containing the rareearth $\mathrm{Eu}[102,123,129]$ or spreading SiC nano-crystals [122] over the device. The strong temperature dependence of the fluorescence intensities excited by ultraviolet irradiation is used to display the temperature distribution at the device surface. The distribution of the fluorescence intensity is magnified by a microscope and monitored by either a (Peltier-cooled) CCD or CMOS camera. The observed fluorescence intensities under a bias are converted into local temperatures using calibration data obtained at a uniform temperature without an electrical bias on the device.

The temperature image of a mesa surface reveals a highly inhomogeneous temperature distribution with a hotspot around the centre of the mesa. Benseman et al revealed that a faint hotspot with a maximum local temperature of $\sim T_{c}$ appears at the centre of the mesa in the low-bias emission regime, and a more significant and oscillatory temperature rise is observed at currents much higher than those of the high bias regime. [102]. The results are consistent with solutions of the heat diffusion equation in the model connecting with the thermal bath only through the superconducting substrate [121]. Minami et al. concluded that the temperature inhomogeneity has nothing to do with the emission, although a hotspot is observed in the high-bias regime [122]. Tsujimoto et al. found that the emission intensity is higher for a nonuniform bias configuration with a weaker hotspot as indicated by the blue plot in Fig. 13 (b), and thus the volume fraction of the superconducting component contributes to the emission power $P$ [123]. The difference in the temperature distribution was explained by considering thermal escape through the electrode. For the nonuniform bias, heat elimination through the electrodes is more efficient to decrease the extent of the hotspot than that for the uniform bias, because the hotspot center locates underneath the biasing electrode, which also works for a heat path, for the nonuniform bias, whereas in-between the two electrodes for the uniform bias.

\subsection{Heat manipulation}

To actively manipulate the self heating in the mesa, a novel structure was invented by sandwiching the stack between a pair of substrates. As a result, $\mathrm{THz}$ emission above $77 \mathrm{~K}$ (liquid nitrogen temperature) was realised, and the frequencies were increased to 

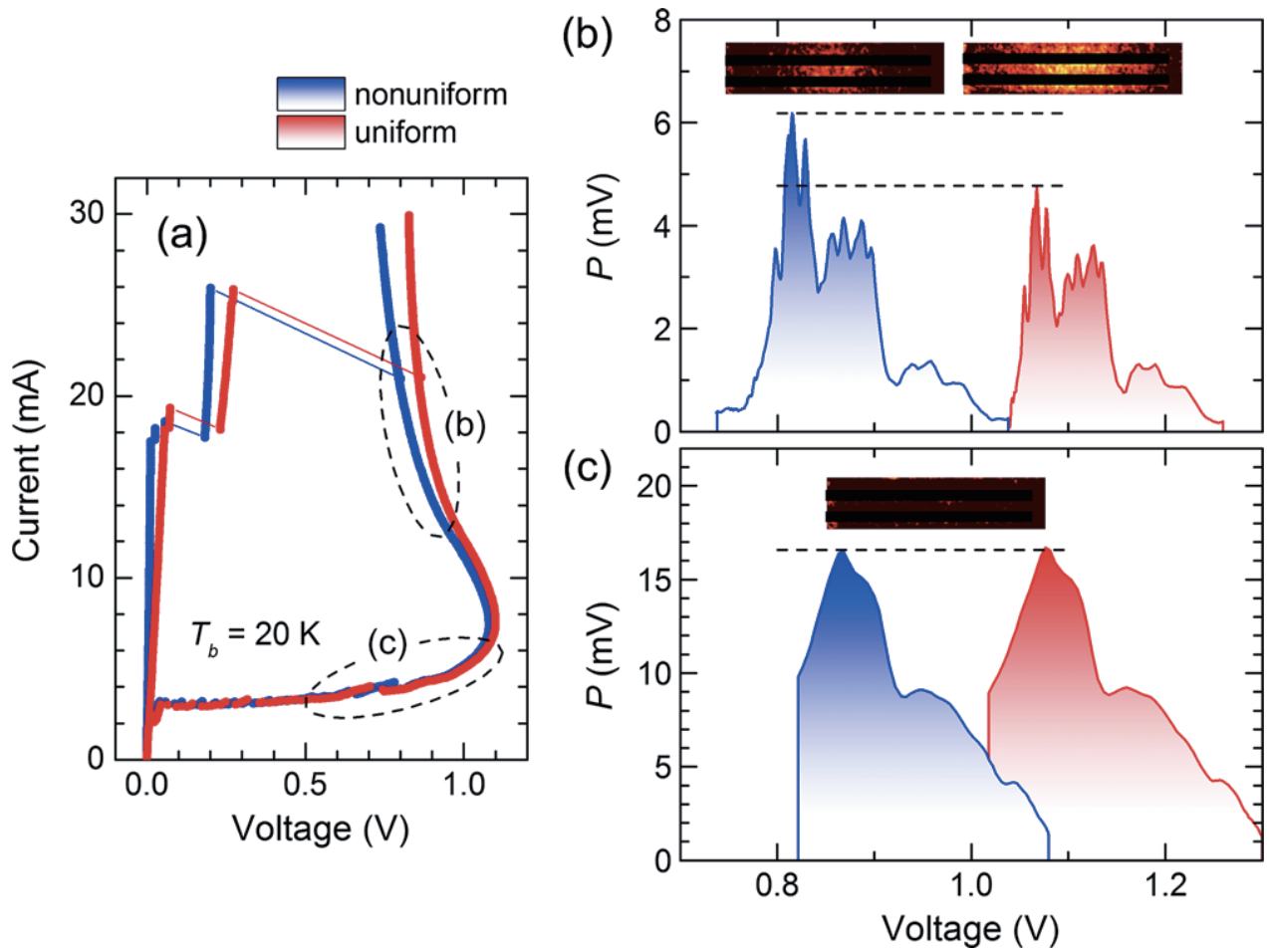

Figure 13. (a) $I-V$ characteristics of a device for nonuniform (bias current is injected through one electrode) and uniform bias (bias current is injected through two electrodes) at $T_{b}=20 \mathrm{~K}$. Bolometer responses $P$ as functions of bias voltage $V$ in the high- and the low-bias regime are shown in (b) and (c), respectively, where the blue and the red plots indicate the data for nonuniform and uniform bias. $\mathrm{V}$ for the latter case has offsets of $0.2 \mathrm{~V}$. The insets above the data present the corresponding temperature distributions in the mesa. (after [123])

$1.05 \mathrm{THz}$ as shown in Fig. 14 [32]. In subsequent experiments, the frequencies were increased to 1.6 and $2.4 \mathrm{THz}$ with a similar sample configuration[33, 34].

Wang et al. started by fabrication of a Bi2212 stack embedded between two gold layers using a previously reported process [130]. The GBG structure sample emits in the range of $0.43-0.82 \mathrm{THz}$. They then glued a second, thermally anchored substrate onto the sample surface (SWS structure). The maximum voltage of this better cooled and dimensionally-unchanged sample increased [32]. Thus, both the emission frequencies and tunable frequency range were significantly increased to 1.05 $\mathrm{THz}$ and $0.71 \mathrm{THz}$, respectively. Sketches of the GBG structure are shown in Fig. 4(a) and (c) and sketches of the SWS structure are shown in Fig. 4(b) and (d). The fabrication details are described in Ref. [32].

The frequency ranges of detectable emission of both the GBG structure and the SWS with respect to $T_{b}$ and $V$ are summarised in Fig. 14(a). Both structures exhibit the same tendency, namely, the emission frequencies gradually increase with decreasing $T_{b}$. The GBG structure was found to radiate emission at several values of $T_{b}$ s below $65 \mathrm{~K}$, whereas the emission from the SWS can be detected over a broader temperature 

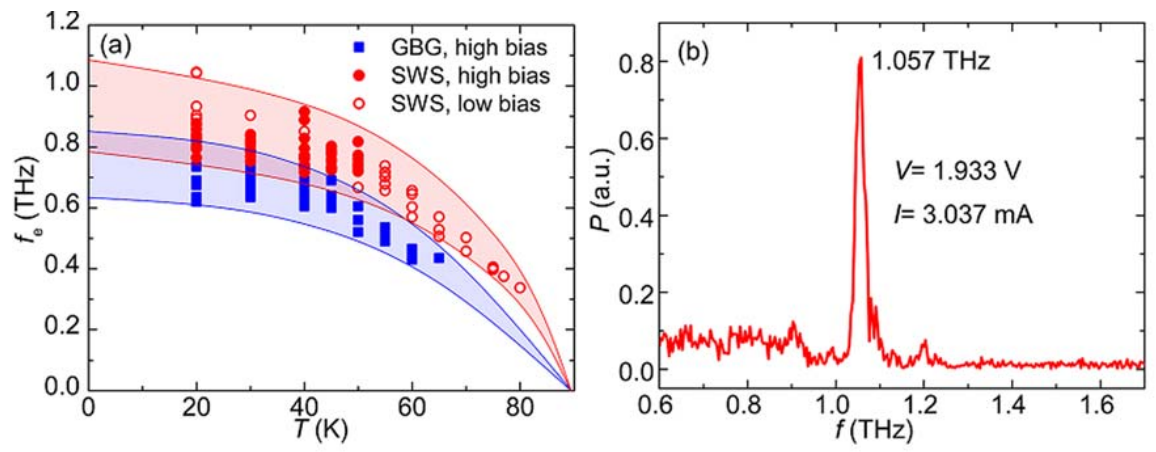

Figure 14. Emission frequency $f_{e}$ vs. (a) the $T_{b}$. (b) shows the spectrum of THz emission at $T_{b}=20 \mathrm{~K}$ and $I=3.037 \mathrm{~mA}$. (after [32]).

range from 20 to $80 \mathrm{~K}$, indicating that the operating temperature can be in the liquid nitrogen temperature range. For the entire temperature regime the SWS exhibited an emission frequency range $(0.34-1.05 \mathrm{THz})$ that is much broader than that of the GBG structure $(0.43-0.82 \mathrm{THz})$. Note that for the SWS emission above $50 \mathrm{~K}$ was observed in the low-bias regime. In addition, the highest $f_{e}$ of $1.05 \mathrm{THz}$, for which the corresponding spectrum is shown in Fig. 14(b), was observed in the low-bias regime of the SWS. Figure 15 shows the typical spectra emission at 1.6 and $2.4 \mathrm{THz}$, as reported in Refs. [33] and [34]. Their expansions of the upper limit of emission frequency were achieved by attaching sapphire substrates both sides of mesas without superconducting substrates (stand-alone samples) [111].

Kakeya et al. argued that an electrode $400 \mathrm{~nm}$ in thickness suppresses the emission because the temperature distribution is too homogenised to induce the synchronization of spatial phase difference [99]. The electrode shape (impedance) is also relevant to the emission properties because a stripline that consists of two electrodes and a mesa possibly carries the excited high-frequency power to the ground. The strong frequency dependence of $P$ is presumably related to the frequency dependence of the transmission loss of the stripline. Electrodes designed for optimising both the heat escape and emission efficiency are the next concern for IJJ THz sources.

A practical method of realising intense $\mathrm{THz}$ emission from IJJs using external local-heating was proposed and demonstrated theoretically[58]. An artificial temperature distribution induced by local heating strongly excites Josephson plasma waves inside IJJs. Accordingly, $P_{t o t}$ of the $\mathrm{THz}$ waves is enhanced dramatically, and can reach on the order of milliwats. Zhou et al. [126] reportedly tuned the $\mathrm{THz}$ emission of a Bi2212 IJJ stack by a focused laser beam that was scanned across the stack. The emission power $P$ increased by up to $75 \%$ upon laser irradiation at a $T_{b}$ near $22 \mathrm{~K}$. The laser-induced changes in the voltage $V$ across the stack and in $P$ were measured simultaneously. The maximum laser-induced change in the emission power $\Delta P$, was achieved by irradiation from a position where the local temperature is approximately equal to the critical temperature $T_{c}$. However, $\Delta P$ was found to be proportional to the laser-induced global voltage change $\Delta V$ irrespective of the laser position. This unexpected global response is likely to be related to a change in the average stack temperature and is consistent with the change in $P$ when $T_{b}$ is increases by about $0.2 \mathrm{~K}$. This tuning method can be employed in the application of Bi2212 

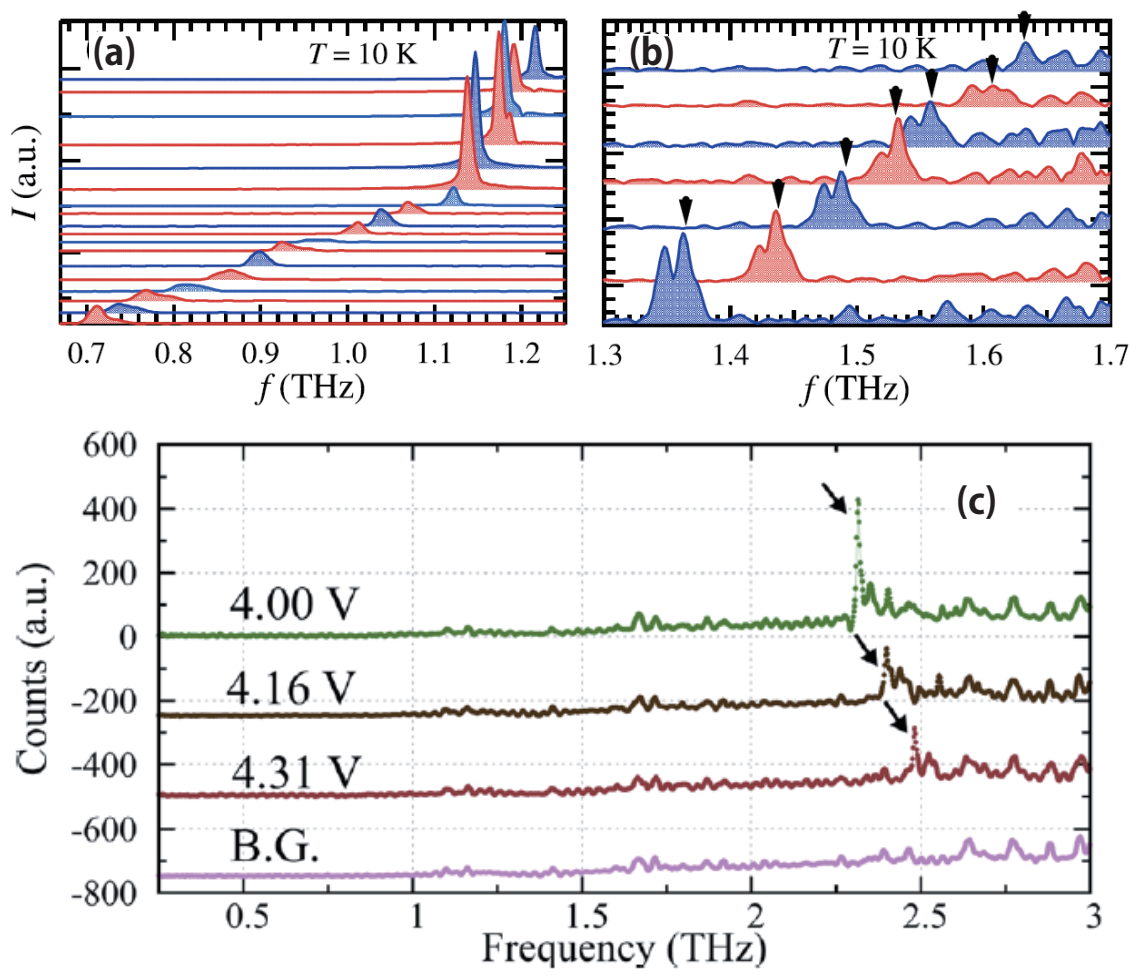

Figure 15. Spectra of $\mathrm{THz}$ emission from $(\mathrm{a}, \mathrm{b})$ a rectangular mesa emitting up to $1.6 \mathrm{THz}$, and (c) a disk mesa emitting up to2.4 THz. Both are stand-alone samples sandwitched by sapphire substrates. (after [33] and [34])

THz sources. Watanabe et al. [131] also observed that when the hotspot position was moved to a mesa end by locally heating the mesa surface with a laser beam, the intensity of the emission increased, but no changes in its frequency or line width were observed. These results suggest that higher power radiation is attainable by adjusting the hotspot position. Note that the beam size here is about $80 \mu \mathrm{m}$ in diameter; this is much larger than $1-2 \mu \mathrm{m}$, which was the typical value in Zhou et al.'s experiment.

\section{Examples of applications of terahertz EM wave sources}

This section describes practical applications of IJJ THz sources. To date, IJJ THz sources have been tested for nondestructive imaging. THz emission above $77 \mathrm{~K}$ allows the use of compact configurations of an IJJ THz source cooled by liquid nitrogen.

$\mathrm{THz}$ waves can be transmitted through paper objects because of their submillimetre wavelength. One applications of $\mathrm{THz}$ waves is the inspectin of the contents inside packages and personal belongings hidden inside clothing. Tsujimoto et al. demonstrated that an IJJ THz source, like other THz sources, can be used for transmission imaging [90]. They provided transmission images of coins inside an envelope and a dandelion leaf. Interference fringes shown in the transmission image also illustrate the monochromaticity of the emitted $\mathrm{THz}$ waves. Kashiwagi et al. 
have presented a reflection image of coins and a computed tomographic image of a three-dimensional object [132, 133].

Portability is one of the most important properties affecting practicality. The recent demonstrations of IJJ THz source above $77 \mathrm{~K}[134,135]$ allow us to realise a compact $\mathrm{THz}$ source consisting of a battery and liquid nitrogen. The recent development of a Stirling cryocooler [136], which is compact and requires no external compressor, enables an all-in-one portable system of high- $T_{c}$ devices operated above $40 \mathrm{~K}$. The IJJ THz source is one of the best applications of the Stirling cryocooler because the performance of the IJJ THz source is optimised in the temperature range between 40 and $60 \mathrm{~K}$. Reductions in the price of the Stirling cryocooler are quite an important factor for broad adoption of IJJ THz sources.

The emitted $\mathrm{THz}$ waves are expected to be coherent because the emission is driven by the synchronization of the stacked IJJs. The coherence, nevertheless, has to be demonstrated in an more explicit manner. High coherence is a least requirement for use of the IJJ THz source as a source of carrier wave for high-speed data transfers such as uncompressed high-resolution (for example, $4 \mathrm{~K}$ resolution) movie files. To this end, we should be able to modulate the emitted $\mathrm{THz}$ waves. The use for local oscillators of superconducting mixers employed for astronomical observations [137] is one of the best promising applications because another cooling system is not required. For mobile communications, the generation of circularly polarised waves is important. Because an IJJ stack functions as a patch antenna, circularly polarised emission can be obtained by changing the mesa shape [138]. This is also an advantage of our IJJ $\mathrm{THz}$ sources. Finally, the circularly polarised $\mathrm{THz}$ waves can also be used in circular dichroism spectroscopy to identify harmful chemicals.

\section{Summary}

The properties of IJJ THz sources are described in this review. These sources possess an extremely broad frequency tuning range of more than $30 \%$ of the central frequency. This remarkable advantage is considered to be attributed to the effective changes in propagation velocity of the excited Josephson plasma oscillation and geometries of the device, both of which are caused by peculiar properties of superconductivity such as strong temperature dependence of the penetration depth and the low thermal conductivity. A large part of the emission frequency range covers a temperature region of $k_{B} T>h f_{e}$, where CW-QCLs are disable to work, with a higher emission power than RTD oscillators. In addition to this, expected high coherence of emitted EM waves is also attributed to superconductivity as a macroscopic quantum phenomenon in solids. The ease of device preparation from a Bi2212 single crystal provides great potential for expanding research opportunities and promoting the use of the device for general purposes such as high-speed telecommunications and nondestructive spectroscopy. Thermal management is found to be an important factor for increasing the emission power and frequency of IJJ THz sources. Another important element is the electrode, which also works as an antenna. The performance of IJJ THz sources must be improved greatly by optimising the thermal management and impedance matching with the space. 


\section{Acknowledgements}

The authors acknowledge H. Asai, T. Benseman, A. Elarabi, T. Hatano, X. Hu, K. Kadowaki, T. Kashiwagi, V. Koshelets, A. Koshelev, R. Kleiner, R. Klemm, T. Koyama, M. Machida, H. Matsumoto, H. Minami, Y. Ota, L. Ozyuzer, M. Suzuki, M. Tachiki, M. Tsujimoto, U. Welp, and T. Yamamoto. This work was supported by KAKENHI (Grant Nos. 26286006 and 25289108).

\section{References}

[1] Josephson B D 1962 Phys. Lett. 1251

[2] Fiske M D 1964 Rev. Mod. Phys. 36 221-222

[3] Anderson P 1964 Special Effects in Superconductivity Lect. Many-body Probl. vol 2 ed Caianiello E R (New York: Elsevier) pp 113-135

[4] Yanson I K, Svistunov V M and Dmitrenko I M 1965 Sov. Phys. JETP 21650

[5] Langenberg D N, Scalapino D J, Taylor B N and Eck R E 1965 Phys. Rev. Lett. 15294

[6] Barbara P, Cawthorne A, Shitov S and Lobb C 1999 Phys. Rev. Lett. 82 1963-1966

[7] Kleiner R, Steinmeyer F, Kunkel G and Müller P 1992 Phys. Rev. Lett. 68 2394-2397

[8] Yurgens A A 2000 Supercond. Sci. Technol. 13 R85-R100 ISSN 0953-2048

[9] Sakai S, Bodin P and Pedersen N F 1993 J. Appl. Phys. 732411

[10] Koyama T and Tachiki M 1996 Phys. Rev. B 5416183

[11] Machida M, Koyama T and Tachiki M 1999 Phys. Rev. Lett. 83 4618-4621 ISSN 0031-9007

[12] Suzuki M, Watanabe T and Matsuda A 1999 Phys. Rev. Lett. 82 5361-5364

[13] Tamasaku K, Nakamura Y and Uchida S 1992 Phys. Rev. Lett. 69 1455-1458

[14] Tsui O, Ong N, Matsuda Y, Yan Y and Peterson J 1994 Phys. Rev. Lett. 73 724-727

[15] Bulaevskii L N, Maley M P and Tachiki M 1995 Phys. Rev. Lett. 74 801-804 ISSN 0031-9007

[16] Matsuda Y, Gaifullin M B, Kumagai K, Kadowaki K and Mochiku T 1995 Phys. Rev. Lett. 75 $4512-4515$

[17] Koshelev A E 1996 Phys. Rev. Lett. 773901

[18] Kakeya I, Kindo K, Kadowaki K, Takahashi S and Mochiku T 1998 Phys. Rev. B 57 3108-3115

[19] Gaifullin M B, Matsuda Y, Chikumoto N, Shimoyama J, Kishio K and Yoshizaki R 1999 Phys. Rev. Lett. 83 3928-3931 ISSN 0031-9007

[20] Sato M, Shibauchi T, Ooi S, Tamegai T and Konczykowski M 1997 Phys. Rev. Lett. 79 37593762 ISSN 0031-9007

[21] Hanaguri T, Tsuchiya Y, Sakamoto S, Maeda A and Steel D 1997 Phys. Rev. Lett. 78 3177-3180 ISSN 0031-9007

[22] Shibauchi T, Nakano T, Sato M, Kisu T, Kameda N, Okuda N, Ooi S and Tamegai T 1999 Phys. Rev. Lett. 83 1010-1013

[23] Gaifullin M B, Matsuda Y, Chikumoto N, Shimoyama J and Kishio K 2000 Phys. Rev. Lett. 842945

[24] Ooi S, Mochiku T and Hirata K 2002 Phys. Rev. Lett. 89247002 ISSN 0031-9007

[25] Krasnov V M, Yurgens A and Winkler D 1999 Phys. Rev. B 59 8463-8466

[26] Kim S M, Wang H B, Hatano T, Urayama S, Kawakami S, Nagao M, Takano Y, Yamashita T and Lee K 2005 Phys. Rev. B $\mathbf{7 2} 140504$

[27] Ustinov A V and Pedersen N F 2005 Phys. Rev. B 72052502 ISSN 1098-0121

[28] Kakeya I, Hamada K, Tachiki T, Watanabe T and Suzuki M 2009 Supercond. Sci. Technol. 22 114014

[29] Ozyuzer L, Koshelev a E, Kurter C, Gopalsami N, Li Q, Tachiki M, Kadowaki K, Yamamoto T, Minami H, Yamaguchi H, Tachiki T, Gray K E, Kwok W K and Welp U 2007 Science 318 1291-1293

[30] Benseman T M, Koshelev a E, Kwok W K, Welp U, Kadowaki K, Cooper J R and Balakrishnan G 2013 Supercond. Sci. Technol. 26085016

[31] An D Y, Yuan J, Kinev N, Li M Y, Huang Y, Ji M, Zhang H, Sun Z L, Kang L, Jin B B, Chen J, Li J, Gross B, Ishii A, Hirata K, Hatano T, Koshelets V P, Koelle D, Kleiner R, Wang H B, Xu W W and Wu P H 2013 Appl. Phys. Lett. 10292601

[32] Ji M, Yuan J, Gross B, Rudau F, An D Y, Li M Y, Zhou X J, Huang Y, Sun H C, Zhu Q, Li J, Kinev N, Hatano T, Koshelets V P, Koelle D, Kleiner R, Xu W W, Jin B B, Wang H B and Wu P H 2014 Appl. Phys. Lett. 105122602

[33] Kashiwagi T, Yamamoto T, Kitamura T, Asanuma K, Watanabe C, Nakade K, Yasui T, Saiwai 
Y, Shibano Y, Kubo H, Sakamoto K, Katsuragawa T, Tsujimoto M, Delfanazari K, Yoshizaki R, Minami H, Klemm R A and Kadowaki K 2015 Appl. Phys. Lett. 106092601

[34] Kashiwagi T, Sakamoto K, Kubo H, Shibano Y, Enomoto T, Kitamura T, Asanuma K, Yasui T, Watanabe C, Nakade K, Saiwai Y, Katsuragawa T, Tsujimoto M, Yoshizaki R, Yamamoto T, Minami H, Klemm R A and Kadowaki K 2015 Appl. Phys. Lett. 107082601 ISSN 0003-6951

[35] Kumar S, Williams B S, Hu Q and Reno J L 2006 Appl. Phys. Lett. 88121123 ISSN 00036951

[36] Walther C, Scalari G, Faist J, Beere H and Ritchie D 2006 Appl. Phys. Lett. 89231121 ISSN 00036951

[37] Walther C, Fischer M, Scalari G, Terazzi R, Hoyler N and Faist J 2007 Appl. Phys. Lett. 91 131122 ISSN 00036951

[38] Scalari G, Walther C, Fischer M, Terazzi R, Beere H, Ritchie D and Faist J 2009 Laser Photonics Rev. 3 45-66 ISSN 18638880

[39] Scalari G, Turcinkova D, Lloyd-Hughes J, Amanti M I, Fischer M, Beck M and Faist J 2010 Appl. Phys. Lett. 97081110 ISSN 00036951

[40] Sushil Kumar S K 2011 Chinese Opt. Lett. 9 110003-110009 ISSN 1671-7694

[41] Kumar S, Chan C W I, Hu Q and Reno J L 2011 Nat. Phys. 7 166-171 ISSN 1745-2473

[42] Reddy M, Martin S, Molnar A, Muller R, Smith R, Siegel P, Mondry M, Rodwell M, Kroemer $\mathrm{H}$ and Allen S 1997 IEEE Electron Device Lett. 18 218-221 ISSN 0741-3106

[43] Suzuki S, Teranishi A, Hinata K, Asada M, Sugiyama H and Yokoyama H 2009 Appl. Phys. Express 2054501 ISSN 1882-0778

[44] Kanaya H, Shibayama H, Sogabe R, Suzuki S and Asada M 2012 Appl. Phys. Express 5124101 ISSN 1882-0778

[45] Kanaya H, Sogabe R, Maekawa T, Suzuki S and Asada M 2014 J. Infrared, Millimeter, Terahertz Waves 35 425-431

[46] Tonouchi M 2007 Nat. Photonics $197-105$

[47] Faist J, Capasso F, Sivco D L, Sirtori C, Hutchinson a L and Cho a Y 1994 Science 264 $553-556$

[48] Köhler R, Tredicucci A, Beltram F, Beere H E, Linfield E H, Davies A G, Ritchie D A, Iotti R C and Rossi F 2002 Nature 417 156-159

[49] Williams B S 2007 Nat. Photonics 1 517-525 ISSN 1749-4885

[50] Sirtori C, Barbieri S and Colombelli R 2013 Nat. Photonics 7 691-701 ISSN 1749-4885

[51] Wade A, Fedorov G, Smirnov D, Kumar S, Williams B S, Hu Q and Reno J L 2009 Nat. Photonics 3 41-45 ISSN 1749-4885

[52] Brown E R, Soderstrom J R, Parker C D, Mahoney L J, Molvar K M and McGill T C 1991 Appl. Phys. Lett. 582291 ISSN 00036951

[53] Hu X and Lin S Z 2010 Supercond. Sci. Technol. 23053001 ISSN 0953-2048 (Preprint $0911.5371)$

[54] Wiesenfeld K, Colet P and Strogatz S 1996 Phys. Rev. Lett. 76 404-407

[55] Watts D J and Strogatz S H 1998 Nature 393 440-442

[56] Welp U, Kadowaki K and Kleiner R 2013 Nat. Photonics 7 702-710

[57] Koyama T, Matsumoto H, Machida M and Ota Y 2011 Supercond. Sci. Technol. 24085007

[58] Asai H and Kawabata S 2014 Appl. Phys. Lett. 104112601

[59] Shukrinov Y M, Rahmonov I R, Plecenik A, Seidel P, Ilichev E and Nawrocki W 2014 Supercond. Sci. Technol. 27124007 ISSN 0953-2048 (Preprint 1408.1541)

[60] Lin S Z 2014 J. Appl. Phys. 115173901 ISSN 0021-8979 (Preprint arXiv:1401.2271v3)

[61] Liu F and Hu X 2015 J. Phys. Soc. Japan 84064719 ISSN 0031-9015

[62] Kadowaki K, Kakeya I, Gaifullin M B, Mochiku T, Takahashi S, Koyama T and Tachiki M 1997 Phys. Rev. B 56 5617-5621 ISSN 0163-1829

[63] Matsuda Y, Gaifullin M B, Kumagai K i, Kosugi M and Hirata K 1997 Phys. Rev. Lett. 78 1972-1975 ISSN 0031-9007

[64] Kosugi M, Matsuda Y, Gaifullin M, Bulaevskii L, Chikumoto N, Konczykowski M, Shimoyama J, Kishio K, Hirata K and Kumagai K 1997 Phys. Rev. Lett. 79 3763-3766 ISSN 0031-9007

[65] Koshelev A E, Bulaevskii L N and Maley M P 1998 Phys. Rev. Lett. 81 902-905 ISSN 0031-9007

[66] Koshelev A E, Bulaevskii L N and Maley M P 2000 Phys. Rev. B 62 14403-14418 ISSN 0163-1829

[67] Kakeya I, Wada T, Kadowaki K and Machida M 2002 Phys. C Supercond. 378-381 437-442 ISSN 09214534

[68] Koyama T 2003 Phys. Rev. B 68224505 ISSN 0163-1829

[69] Kakeya I, Wada T, Nakamura R and Kadowaki K 2005 Phys. Rev. B - Condens. Matter Mater. Phys. 72 14540-14541

[70] Machida M, Koyama T, Tanaka A and Tachiki M 2000 Physica C 330 85-93 
[71] Tachiki M, Iizuka M, Minami K, Tejima S and Nakamura H 2005 Phys. Rev. B - Condens. Matter Mater. Phys. $\mathbf{7 1} 134515$

[72] Lin S, Hu X and Tachiki M 2008 Phys. Rev. B 77014507 ISSN 1098-0121 (Preprint 0709.0580)

[73] Kakeya I, Yamzaki T, Kohri M, Yamamoto T and Kadowaki K 2006 Phys. C Supercond. 437 $118-121$

[74] Wang H B, Urayama S, Kim S M, Arisawa S, Hatano T and Zhu B Y 2006 Appl. Phys. Lett. 89 58-61 ISSN 00036951

[75] Batov I E, Jin X Y, Shitov S V, Koval Y, Muüller P and Ustinov A V 2006 Appl. Phys. Lett. 88262504

[76] Bae M H, Lee H J and Choi J H 2006 Phys. Rev. Lett. 9827002

[77] Kawayama I, Zhang C, Wang H and Tonouchi M 2013 Supercond. Sci. Technol. 26093002 ISSN 0953-2048

[78] Tsujimoto M, Yamaki K, Deguchi K, Yamamoto T, Kashiwagi T, Minami H, Tachiki M, Kadowaki K and Klemm R A 2010 Phys. Rev. Lett. 10537005

[79] Delfanazari K, Asai H, Tsujimoto M, Kashiwagi T, Kitamura T, Ishida K, Watanabe C, Sekimoto S, Yamamoto T, Minami H, Tachiki M, Klemm R A, Hattori T and Kadowaki K $2014 \mathrm{~J}$. Infrared, Millimeter, Terahertz Waves 35 131-146 ISSN 1866-6892

[80] Delfanazari K, Asai H, Tsujimoto M, Kashiwagi T, Kitamura T, Yamamoto T, Wilson W, Klemm R A, Hattori T and Kadowaki K 2015 IEEE Trans. Terahertz Sci. Technol. 5 505511 ISSN 2156-342X

[81] Nishikata T, Kato T, Kotaki Y, Suematsu H, Kawakami A and Yasui K 2014 Jpn. J. Appl. Phys. 53 04EJ02 ISSN 0021-4922

[82] Vopilkin E A, Chiginev A V, Revin L S, Tropanova A N, Shuleshova I Y, Okhapkin A I, Shovkun A D, Kulakov A B and Pankratov A L 2015 Supercond. Sci. Technol. 28045006 ISSN 0953-2048

[83] Ozyuzer L, Simsek Y, Koseoglu H, Turkoglu F, Kurter C, Welp U, Koshelev A E, Gray K E, Kwok W K, Yamamoto T, Kadowaki K, Koval Y, Wang H B and Müller P 2009 Supercond. Sci. Technol. 22114009 ISSN 0953-2048

[84] Koseoglu H, Turkoglu F, Simsek Y and Ozyuzer L 2011 J. Supercond. Nov. Magn. 24 1083-1086 ISSN 1557-1939

[85] Orita N, Minami H, Koike T, Yamamoto T and Kadowaki K 2010 Phys. C Supercond. its Appl. 470 S786-S787

[86] Tsujimoto M, Yamamoto T, Delfanazari K, Nakayama R, Kitamura T, Sawamura M, Kashiwagi T, Minami H, Tachiki M, Kadowaki K and Klemm R A 2012 Phys. Rev. Lett. 108107006

[87] Yuan J, Li M Y, Li J, Gross B, Ishii A, Yamaura K, Hatano T, Hirata K, Takayama-Muromachi E, Wu P H, Koelle D, Kleiner R and Wang H B 2012 Supercond. Sci. Technol. 25075015 ISSN 0953-2048

[88] Nakajima K et al. 2014 Successful terahertz emission from monolithic Bi-2212 intrinsic Josephson junctions near below $77 \mathrm{~K}$ abstract of THz-plasma2014

[89] Minami H, Kakeya I, Yamaguchi H, Yamamoto T and Kadowaki K 2009 Appl. Phys. Lett. 95 232511

[90] Tsujimoto M, Minami H, Delfanazari K, Sawamura M, Nakayama R, Kitamura T, Yamamoto T, Kashiwagi T, Hattori T and Kadowaki K 2012 J. Appl. Phys. 111123111

[91] Turkoglu F, Koseoglu H, Demirhan Y, Ozyuzer L, Preu S, Malzer S, Simsek Y, Müller P, Yamamoto T and Kadowaki K 2012 Supercond. Sci. Technol. 25125004 ISSN 0953-2048

[92] Oikawa D, Irie A, Yamaki K and Oya G i 2012 Phys. Procedia 27 312-315 ISSN 18753892

[93] Eisele H, Naftaly M and Fletcher J R 2007 Meas. Sci. Technol. 182623

[94] Wang H B, Guénon S, Gross B, Yuan J, Jiang Z G, Zhong Y Y, Grünzweig M, Iishi A, Wu P H, Hatano T, Koelle D and Kleiner R 2010 Phys. Rev. Lett. 105057002

[95] Kakeya I, Hirayama N, Nakagawa T, Omukai Y and Suzuki M 2013 Phys. C Supercond. its Appl. 491 11-15

[96] Kashiwagi T, Tsujimoto M, Yamamoto T, Minami H, Yamaki K, Delfanazari K, Deguchi K, Orita N, Koike T, Nakayama R, Kitamura T, Sawamura M, Hagino S, Ishida K, Ivanovic K, Asai H, Tachiki M, Klemm R A and Kadowaki K 2012 Jpn. J. Appl. Phys. 51010113

[97] Li M, Yuan J, Kinev N, Li J, Gross B, Guénon S, Ishii A, Hirata K, Hatano T, Koelle D, Kleiner R, Koshelets V, Wang H and Wu P 2012 Phys. Rev. B 8660505

[98] Kadowaki K, Yamaguchi H, Kawamata K, Yamamoto T, Minami H, Kakeya I, Welp U, Ozyuzer L, Koshelev A, Kurter C, Gray K E and Kwok W K 2008 Phys. C Supercond. its Appl. 468 634-639

[99] Kakeya I, Omukai Y, Yamamoto T, Kadowaki K and Suzuki M 2012 Appl. Phys. Lett. 100 242603 
[100] Wang H B, Guénon S, Yuan J, Iishi A, Arisawa S, Hatano T, Yamashita T, Koelle D and Kleiner R 2009 Phys. Rev. Lett. 10217006

[101] Omukai Y, Kakeya I and Suzuki M 2012 J. Phys. Conf. Ser. 400052027

[102] Benseman T M, Koshelev A E, Kwok W K, Welp U, Vlasko-Vlasov V K, Kadowaki K, Minami H and Watanabe C 2013 J. Appl. Phys. 113133902

[103] Tsujimoto M, Kakeya I, Kashiwagi T, Minami H and Kadowaki K 2016 Opt. Express 244591 ISSN 1094-4087 (Preprint 1509.02985)

[104] Benseman T, Koshelev A, Gray K, Kwok W K, Welp U, Kadowaki K, Tachiki M and Yamamoto T 2011 Phys. Rev. B 84064523

[105] Kakeya I, Hirayama N, Omukai Y and Suzuki M 2015 J. Appl. Phys. 117043914

[106] Benseman T M, Gray K E, Koshelev A E, Kwok W K, Welp U, Minami H, Kadowaki K and Yamamoto T 2013 Appl. Phys. Lett. 103022602

[107] Sekimoto S, Watanabe C, Minami H, Yamamoto T, Kashiwagi T, Klemm R A and Kadowaki K 2013 Appl. Phys. Lett. 103182601

[108] Yamaki K, Tsujimoto M, Yamamoto T, Furukawa A, Kashiwagi T, Minami H and Kadowaki K 2011 Opt. Express 19 3193-3201

[109] Klemm R a and Kadowaki K 2010 J. Phys. Condens. Matter 22375701

[110] Kitamura T, Kashiwagi T, Yamamoto T, Tsujimoto M, Watanabe C, Ishida K, Sekimoto S, Asanuma K, Yasui T, Nakade K, Shibano Y, Saiwai Y, Minami H, Klemm R A and Kadowaki K 2014 Appl. Phys. Lett. 105202603

[111] Kashiwagi T, Yamamoto T, Minami H, Tsujimoto M, Yoshizaki R, Delfanazari K, Kitamura T, Watanabe C, Nakade K, Yasui T, Asanuma K, Saiwai Y, Shibano Y, Enomoto T, Kubo H, Sakamoto K, Katsuragawa T, Marković B, Mirković J, Klemm R A and Kadowaki K 2015 Phys. Rev. Appl. 4054018 ISSN 2331-7019

[112] Tallon J L, Bernhard C, Shaked H, Hitterman R L and Jorgensen J D 1995 Phys. Rev. B 51 12911-12914

[113] Turkoglu F, Ozyuzer L, Koseoglu H, Demirhan Y, Preu S, Malzer S, Simsek Y, Wang H and Muller P 2013 Phys. C Supercond. 491 7-10 ISSN 09214534

[114] Tsujimoto M, Maeda Y, Kambara H, Elarabi A, Yoshioka Y, Nakagawa Y, Wen Y, Doi T, Saito H and Kakeya I 2015 Supercond. Sci. Technol. 28105015 ISSN 0953-2048

[115] Tajima S, Gu G D, Miyamoto S, Odagawa A and Koshizuka N 1993 Phys. Rev. B $4816164-$ 16167

[116] Shibata H and Matsuda A 1999 Phys. Rev. B 59 R11672-R11674 ISSN 0163-1829

[117] Motohashi T, Shimoyama J, Kitazawa K, Kishio K, Kojima K, Uchida S and Tajima S 2000 Phys. Rev. B 61 R9269-R9272

[118] Shibauchi T and Horiuchi S 2007 Phys. C Supercond. its Appl. 460-462 174-177 ISSN 09214534

[119] Gaifullin M, Matsuda Y, Saito Y and Hirata K 2001 Phys. C Supercond. 357-360 520-522 ISSN 09214534

[120] Thorsmø lle V K, Averitt R D, Maley M P, Bulaevskii L N, Helm C and Taylor A J 2001 Opt. Lett. 261292 ISSN 0146-9592

[121] Yurgens A 2011 Phys. Rev. B 83184501

[122] Minami H, Watanabe C, Sato K, Sekimoto S, Yamamoto T, Kashiwagi T, Klemm R A and Kadowaki K 2014 Phys. Rev. B - Condens. Matter Mater. Phys. 8954503

[123] Tsujimoto M, Kambara H, Maeda Y, Yoshioka Y, Nakagawa Y and Kakeya I 2014 Phys. Rev. Appl. 2044016

[124] Rudau F, Tsujimoto M, Gross B, Judd T E, Wieland R, Goldobin E, Kinev N, Yuan J, Huang Y, Ji M, Zhou X J, An D Y, Ishii A, Mints R G, Wu P H, Hatano T, Wang H B, Koshelets V P, Koelle D and Kleiner R 2015 Phys. Rev. B 91104513 ISSN 1098-0121

[125] Rudau F, Wieland R, Langer J, Zhou X J, Ji M, Kinev N, Hao L Y, Huang Y, Li J, Wu P H, Hatano T, Koshelets V P, Wang H B, Koelle D and Kleiner R 2016 3D simulations of the electrothermal and $\mathrm{THz}$ emission properties of $\mathrm{Bi}_{2} \mathrm{Sr}_{2} \mathrm{CaCu}_{2} \mathrm{O}_{8}$ intrinsic Josephson junction stacks (Preprint arXiv:1602.08966)

[126] Zhou X J, Yuan J, Wu H, Gao Z S, Ji M, An D Y, Huang Y, Rudau F, Wieland R, Gross B, Kinev N, Li J, Ishii A, Hatano T, Koshelets V P, Koelle D, Kleiner R, Wang H B and Wu P H 2015 Phys. Rev. Appl. 3 044012 ISSN 2331-7019

[127] Guénon S, Grünzweig M, Gross B, Yuan J, Jiang Z, Zhong Y, Li M, Iishi A, Wu P, Hatano T, Mints R, Goldobin E, Koelle D, Wang H and Kleiner R 2010 Physical Review B 82214506 ISSN 1098-0121

[128] Gross B, Guénon S, Yuan J, Li M, Li J, Ishii A, Mints R, Hatano T, Wu P, Koelle D, Wang H and Kleiner R 2012 Phys. Rev. B 86094524

[129] Akiyama H, Pyon S, Tamegai T, Tsujimoto M and Kakeya I 2015 Phys. C Supercond. its Appl. 
518 77-80 ISSN 09214534

[130] Wang H, Wu P and Yamashita T 2001 IEEE Transactions on Appiled Superconductivity 11 1199-1202 ISSN 10518223

[131] Watanabe C, Minami H, Kitamura T, Asanuma K, Nakade K, Yasui T, Saiwai Y, Shibano Y, Yamamoto T, Kashiwagi T, Klemm R a and Kadowaki K 2015 Appl. Phys. Lett. 106042603 ISSN 0003-6951

[132] Kashiwagi T, Nakade K, Marković B, Saiwai Y, Minami H, Kitamura T, Watanabe C, Ishida K, Sekimoto S, Asanuma K, Yasui T, Shibano Y, Tsujimoto M, Yamamoto T, Mirković J and Kadowaki K 2014 Appl. Phys. Lett. 104022601

[133] Kashiwagi T, Nakade K, Saiwai Y, Minami H, Kitamura T, Watanabe C, Ishida K, Sekimoto S, Asanuma K, Yasui T, Shibano Y, Tsujimoto M, Yamamoto T, Marković B, Mirković J, Klemm R A and Kadowaki K 2014 Appl. Phys. Lett. 104082603

[134] Hao L Y, Ji M, Yuan J, An D Y, Li M Y, Zhou X J, Huang Y, Sun H C, Zhu Q, Rudau F, Wieland R, Kinev N, Li J, Xu W W, Jin B B, Chen J, Hatano T, Koshelets V P, Koelle D, Kleiner R, Wang H B and Wu P H 2015 Phys. Rev. Appl. 3024006

[135] Minami H, Watanabe C, Kashiwagi T, Yamamoto T, Kadowaki K and Klemm R A $2016 \mathrm{~J}$. Phys. Condens. Matter 28025701 ISSN 0953-8984

[136] Richter H, Greiner-Bär M, Pavlov S G, Semenov A D, Wienold M, Schrottke L, Giehler M, Hey R, Grahn H T and Hübers H W 2010 IRMMW-THz 2010 - 35th Int. Conf. Infrared, Millimeter, Terahertz Waves, Conf. Guid. 18 5890-5896 ISSN 1094-4087

[137] Uzawa Y, Kroug M, Kojima T, Takeda M, Candotti M, Fujii Y, Kaneko K, Shan W, Noguchi T and Wang Z 2009 Supercond. Sci. Technol. 22114002 ISSN 0953-2048

[138] Elarabi A, Yoshioka Y, Tsujimoto M, Nakagawa Y and Kakeya I 2016 Polarization Enhancement of terahertz radiation generated by intrinsic Josephson junctions in a truncated edge square 